\title{
Developmental Analysis of Murine Promyelocyte Leukemia Zinc Finger (PLZF) Gene Expression: Implications for the Neuromeric Model of the Forebrain Organization
}

\author{
Virginia Avantaggiato,, ${ }^{1}$ Pier Paolo Pandolfi, ${ }^{2, a}$ Martin Ruthardt, ${ }^{3}$ Nicola Hawe, ${ }^{2, a}$ Dario Acampora, ${ }^{1}$ Pier \\ Giuseppe Pelicci, ${ }^{3}$ and Antonio Simeone ${ }^{1}$ \\ 'International Institute of Genetics and Biophysics, Consiglio Nazionale delle Ricerche, 80125 Naples, Italy, \\ 2Department of Haematology, Royal Postgraduate Medical School, Hammersmith Hospital, London W12 0NN, \\ United Kingdom, and ${ }^{3}$ sstituto Clinica Medica, Policlinico Monteluce, 06100 Perugia, Italy
}

\begin{abstract}
Promyelocyte Leukemia Zinc Finger ( $P L Z F$ ) is a Kruppet like zinc finger gene previously identified in a unique case of acute promyelocytic leukemia (APL) as the counterpart of a reciprocal chromosomal translocation involving the retinoic acid receptor $\alpha$ gene (RAR $\alpha$ ). PLZF is highly conserved throughout evolution from yeast to mammals. To elucidate its role, we isolated the murine PLZF gene and studied its expression during embryogenesis.

$P L Z F$ is expressed in an extremely dynamic pattern with transcripts appearing at $\mathbf{E} 7.5$ in the anterior neuroepithelium and quickly spreading to the entire neuroectoderm until $E$ 10. At $E$ 8.5, PLZF is transcribed in most of the endoderm.
\end{abstract}

During mid to late gestation $P L Z F$ is expressed in restricted domains of the developing CNS as well as in specific organs and body structures. We have focused our attention on the developing forebrain where $P L Z F$ is transcribed in a transverse, segment-like domain corresponding to the anterior pretectum, in the alarmost part of the dorsal thalamus, in the epithalamus, and in the hypothalamus along a defined longitudinal subdomain. Furthermore, $P L Z F$ is expressed in several segmentary boundaries, among them, the zona limitans intrathalamica. Combined analysis with other regionally restricted genes, such as Orthopedia and $D I x 1$, indicates that in the hypothalamus the $P L Z F$ domain is contained within that of Orthopedia and both are complementary to that of $D / \times 1$.

Our data suggest a role for $P L Z F$ in the establishment and maintenance of transverse identities, longitudinal subdomains, and interneuromeric boundaries, providing additional evidences in favor of the neuromeric organization of the forebrain.

[Key words: PLZF, CNS, forebrain, segmentation, neuromeres, hemopoiesis]

\footnotetext{
Nov. 23, 1994; revised Jan. 30, 1995; Accepted Feb. 2, 1994

We thank L. Luzzatto for helpful discussion and comments on the manuscript. We are grateful to A. Secondulfo and D. Capone for the correction and typing of the Inanuscript. This work was supported by grants from the Telethon Programme and the Italian Association for Cancer Research (A.I.R.C.)

Correspondence should be addressed to Antonio Simeone, International Institute of Genctics and Biophysics, CNR, Via G. Marconi, 12, 80125 Naples, Italy.

Present address: Department of Human Genetics, Memorial Sloan-Kettering Cancer Center, New York, NY.

Copyright (C) 1995 Society for Neuroscience $0270-6474 / 95 / 154927-16 \$ 05.00 / 0$
}

The morphogenesis of the CNS and the differentiation of the neural structures are highly complex processes. The first event during embryonic development is represented by the so-called neural induction. This phenomenon is defined as an interaction between an inducing and a responding tissue, the result of which is a change in the differentiative fate of the latter (Gurdon, 1987). When induced by an organizer (Spemann and Mangold, 1924), responding ectoderm tissue undergoes morphogenetic changes and gives rise to a complete patterned CNS. The phenomenon of regional differentiation in the induced CNS is called regionalization (Gallera, 1971; Hara, 1978; Storey et al., 1992; Ruiz i Altaba, 1993, 1994).

When the neural pattern is established, a complex temporally and spatially regulated series of morphogenetic events (e.g., cell differentiation and migration) gives rise to smaller areas that are phylogenetically, functionally, and often morphogenetically different.

Repeated regions have been interpreted as segment-like structures. This architecture is particularly evident in the hindbrain (Kuhlenbeck, 1973; Puelles et al., 1987; Lumsden and Keynes, 1989; Fraser et al., 1990; Lumsden, 1990).

In the rostral vesicles, the first overall division is followed by a subsequent differentiation of various neuroepithelial domains, resulting in the identification of neural structures with different histologies (Altman and Bayer, 1986, 1988; Puelles et al., 1987).

Anatomical, as well as histological studies, postulate the existence of genetic fate determinants that subdivide the large neural regions into smaller longitudinal and transverse domains (Vaage, 1969; Altman and Bayer, 1988; Bulfone et al., 1993a; Figdor and Stern, 1993). Two main models have carefully described the morphology of the forebrain: the columnar (Herrick, 1933; Kuhlenbeck, 1973) and the neuromeric (Vaage, 1969; Puelles and Rubenstein, 1993; Rubenstein et al., 1994; see also discussion; Kuhlenbeck, 1973) models. Furthermore, the segmental organization of the embryonic diencephalon has been recently proposed (Figdor and Stern, 1993), demonstrating that each segmental unit is represented by a polyclonal cell population with a restricted cell fate.

Specific gene combinations could supply positional and differentiative information to define a regional identity both in the hindbrain as well as in the rostral CNS (revicwed in McGinnis and Krumlauf, 1992; Rubenstein et al., 1994).

Gene candidates for the specification of forebrain regions have been isolated. Several of these are homologs of Drosophila 
genes controlling head development such as empty spiracles (ems), orthodenticle (otd), Distal-less (Dll), and orthopedia ( $\mathrm{Dm}$ otp) (Dalton et al., 1989; Cohen and Jürgens, 1991; Finkelstein and Perrimon, 1991; Porteus et al., 1991; Price et al., 1991, 1992; Simeone et al., 1992a,b, 1993, 1994a,b). In addition, mouse genes related to Drosophila $N K$, Wingless $(\mathrm{Wg})$, engrailed (en) and the Pax gene family have been also isolated and studied (Kim and Nirenberg 1989; Martinez et al., 1991; Roelink and Nusse, 1991; McMahon et al., 1992; Price et al., 1992; Bulfone, 1993; Stoykova and Gruss, 1991). Their expression pattern is more consistent with the longitudinal and transverse boundaries proposed in the neuromeric model (reviewed in Puelles and Rubenstein, 1993; Rubenstein et al., 1994), suggesting the existence of a highly conservative evolutionary law that might regulate the most anterior axial patterning in the embryonic rostral CNS.

Furthermore, many examples do exist, indicating that regulatory genes involved in the establishment of the neural cell identity and phenotype are identical or closely related to those controlling the fate of hemopoietic cells (reviewed in Jessel and Melton, 1992).

On these bases, we report a detailed analysis of the Promyelocyte Leukemia Zinc Finger (PLZF) gene expression during murine embryonic development. Our results suggest that besides in the hemopoiesis, a major role could be played by PLZF also in the early neuroepithelial differentiation, in the establishment of regional identities of forebrain derivatives and in the differentiation of specific body structures and organs.

These data support the neuromeric model and reinforce the idea that a complex combinatorial code of regionally restricted genes, belonging to different regulatory gene families, supply positional as well as differentiative information in the organization of the forebrain.

\section{Materials and Methods}

Isolation of PLZF $c D N A$ and relative probes. Single-stranded cDNAs were generated from $1 \mu \mathrm{g}$ of KG1 total RNA using a commercial kit (Promega RiboClone cDNA Synthesis System; Promega Corporation) and amplified in a standard 40-cycle PCR reaction (Saiki et al., 1988). Denaturing, annealing, and elongation temperatures were $94^{\circ} \mathrm{C}, 60^{\circ} \mathrm{C}$ and $72^{\circ} \mathrm{C}$, respectively. $\mathrm{MgCl}_{2}$ final concentration was $2.5 \mathrm{mM}$. To generate the P6 DNA probe the following primers were used: PLZFI $5^{\prime}$ AAGCCTCATGCCTGAGCCGA- $3^{\prime}$ as the $5^{\prime}$ primer and $P L Z F 25^{\prime}$ TACTCGATCTCCAGGATCTC-3' as $3^{\prime}$ primer. The mouse macrophage cDNA library (purchased from Clontech) was screened according to established procedures (Maniatis) using the P6 probe. The P5 and AS mouse $P L Z F$ probes were generated from one of the isolated mouse PLZF cDNAs by PCR using the following primers: PLZFn4 5'-TTCATCCAGAGGGAGCTGTT- $3^{\prime}$ and PLZFn 5 5'-CACACTCGTAGGGGTGGTCGCCTGTATGTG- $3^{\prime}$ for P5 and $P L Z F-M 5$ 5'-CGAGGAGCCAACTCTGGC-3' and PLZF-M6 5'-GAGCTCGCACCCGTACG-3' for AS.

The PCR amplified fragments were cloned into the pCRII vector using the Invitrogen TA Cloning System (Invitrogen). T7 and Sp6 forward primers present into the pCRII vector were used for sequencing.

Zoo blot analysis. The Zoo blot was prehybridized for $3 \mathrm{hr}$ and hybridized for $18 \mathrm{hr}$ at $65^{\circ} \mathrm{C}$ in $3 \times \mathrm{SSC}, 10 \times$ Denhardt's solution, $10 \%$ Dextran, $100 \mu \mathrm{g} / \mathrm{ml}$ Salmon sperm, $0.1 \%$ SDS. The filter was washed three times ( $30 \mathrm{~min}$ for each wash) at $65^{\circ} \mathrm{C}$ in $3 \times \mathrm{SSC} / 0.1 \%$ SDS and then exposed at $-80^{\circ} \mathrm{C}$ for 12,96 , and $168 \mathrm{hr}$. The DNA probe was ${ }^{32} \mathrm{P}$ labeled by "random priming." For the analysis we used the murine $P L Z F$ AS probe, which spans the alternative exon and first zinc finger

$R T-P C R$. Total mRNA $(1 \mu \mathrm{g})$ was converted to single-stranded cDNA using random primers (cDNA Cycle TM Kit Invitrogen, CA). Each of these cDNAs was amplified with two $P L Z F$-specific primers, $\mathrm{M} 2$ and M1, producing a fragment of 204 base pairs (bp). As a positive control for amplification we used two abl primers, $\mathrm{CA} 3-$ and A2, giving rise to a fragment of $278 \mathrm{bp}$. The sequences of the primers are as follows: M2, 5'-GAGCTGGCTGTGGGCATGAA-3'; M1, 5' $5^{\prime}$ TG $\wedge$ GCCAGTAAGTGCATT-3'; CA3, 5'-TGTTGACTGGCGTGATGTAGTTGCTTGG-3'; A2, 5'-TTCAGCGGCCAGTAGCATCTGACTT$3^{\prime}$. PCR amplifications were carried out in a buffer containing $12 \mathrm{~mm}$ $\mathrm{MgCl}_{2}$ over 40 cycles, after an initial denaturation step at $94^{\circ} \mathrm{C}$ for 5 min. Conditions during the reaction were as follows: denaturation at $94^{\circ} \mathrm{C}$ for $45 \mathrm{sec}$, primer annealing at $55^{\circ} \mathrm{C}$ for $1 \mathrm{~min}$; primer extension at $72^{\circ} \mathrm{C}$ for $1 \mathrm{~min}$.

Synthesis ${ }^{15}$ S-labeled RNA probes for Otp for RNase protection and in situ hybridization. For in situ hybridization experiments, mouse $P L Z F$ sense and antisense RNA probes were synthetically produced by using the murine cDNA fragment corresponding to the probe P5 (Fig. 1). Mouse Otxl, Otp, and Dlxl are the same probes described in Simeone et al. (1993, 1994b).

For in situ hybridization, transcription reactions with T7 or Sp6 polymerase (Riboprobe Kit, Promega Biotec) were carried out in the presence of $\left[{ }^{35} \mathrm{~S}\right] \mathrm{CTP}$ (Amersham). The template was then degraded with RNase-free DNase (Pharmacia) and the labeled RNA was purified through a Sephadex G-50 column. In situ transcripts were progressively degraded by random alkaline hydrolysis to an average length of 150 nucleotides. The probes were dissolved at a working concentration of $1 \times 10^{5} \mathrm{cpm} / \mathrm{ml}$ in the hybridization mix (Wilkinson and Green, 1990).

In situ hybridization. In situ hybridization was carried out as described by Wilkinson and Green (1990), with minor modifications. The appropriate probe $(30 \mathrm{ml})$ in the hybridization mix was added to each slide. Hybridization was carried out overnight at $55^{\circ} \mathrm{C}$. The slides were then washed under stringent conditions $\left(65^{\circ} \mathrm{C}, 2 \times \mathrm{SSC}, 50 \%\right.$ formamide) and treated with RNase to remove any unhybridized and nonspecifically bound probe. Autoradiography was performed with Kodak NT/ B2 emulsion. Exposure times were between 5 and $12 \mathrm{~d}$. After developing, sections were stained in $0.02 \%$ toluidine blue and mounted in De Pex (Serva). Sections were examined and photographed using a Zeiss SV11 microscope with both dark- and bright-field illumination.

$R A$ administration and malformation analysis. In a first preliminary experiment we quantified heterogeneity in embryonic development of several normal litters. Using standard morphological markers according to Theiler staging (Theiler, 1989), a typical CD1 litter consists of $60-$ $70 \%$ of embryos of the expected developmental stage; $20-30 \%$ of embryos 4-8 hr delayed and $10-20 \%$ 4-6 hr anticipated. We then performed the RA administration experiment as follows: CD1 mice were mated between 1600 and $2300 \mathrm{hr}$, then scored for the presence of the vaginal plug; $1200 \mathrm{hr}$ the next day is considered $\mathrm{E} 0.5$. At the corresponding times shown in Figure 10 (E 7.4 and 7.8) all-trans-retinoic acid (Sigma) $(25 \mathrm{mg} / \mathrm{ml}$ in dimethylsulfoxide) was diluted 1:10 in vegetable oil just before use, and $0.2 \mathrm{ml}$ delivered by gavage, for a final dose of $20 \mathrm{mg} / \mathrm{kg}$ of maternal body weight (Conlon and Rossant, 1992; Marshall et al., 1992). Control mice were administered the same mixture without RA. At E 10-E 10.5 RA-administered mice were sacrificed by cervical dislocation and scored for phenotype malformations. A variable number ranging from 250 to 400 embryos for each time of RA administration were scored, showing a very high penetrance $(\sim 90 \%)$ of the phenotype shown in Figure 10 (A. Simeone, V. Avantaggiato, M. C. Moroni, F. Mavilio, C. Arra, F. Cotelli, V. Nigro, and D. Acampora, unpublished observations). Representative members $(n=10)$ of the two different phenotypes were then utilized for in situ hybridization.

\section{Results}

\section{Murine PLZF gene structure and conservation throughout} evolution

A human PLZF cDNA probe was isolated by RT-PCR from KG1 acute myelogenous leukemia RNA (probe P6, Fig. 1a). A mouse macrophage cDNA library was then screened with the P6 probe (see Materials and Methods) and several murine PLZF cDNAs were isolated. The deduced amino acid sequence were compared to the human $P L Z F$ coding sequence, revealing a very high degree of homology $(\sim 96 \%)$ and a full conservation of the structural domains (Fig. 1b) (Chen et al., 1993). It is worth noting the presence of a 100 amino acid domain at the N-terminal region corresponding to the so-called POZ domain (Fig. 1b). This domain has been recently described to mediate proteinprotein interaction (Bardwell and Treisman, 1994).

Two murine $P L Z F$ cDNA probes were then generated from 
a

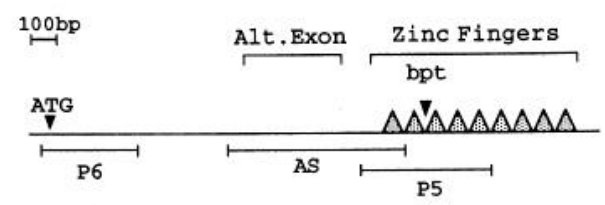

C

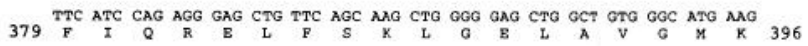

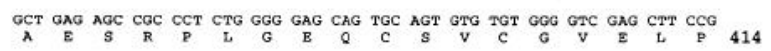

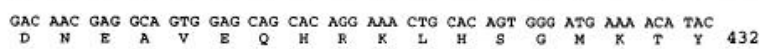

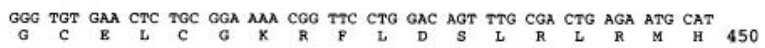

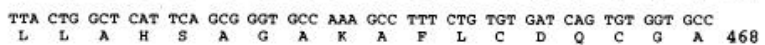

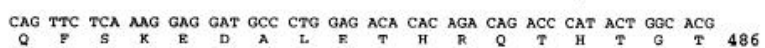

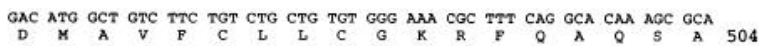

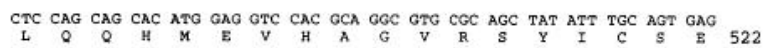

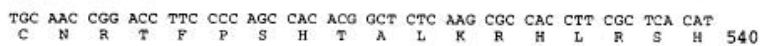
ACA GGC GAC CAC CCC TAC GAG TGT b

MDLTKMGMIQ LQNPSHPTGL LCKANQMRLA GTLCDVVIMV DSQEFHAHRT VLACTSKMFE 60 ILFHRNSQHY TLDFLSPKTF QQILEYAYTA TLQAKAEDLD DLLYAAEILE IEYLEEQCLK 120 ILETIQASDD NDTEATMADG GGEEEDDRKA RYLKNIFISK HSSEESGYAS VAGQSLPGPM 180 VDQSPSVSTS FGLSAMSPTK AAVDSLMSIG QSLLQGTLQP PAGPEEPTLA GGGRHPGVAE 240

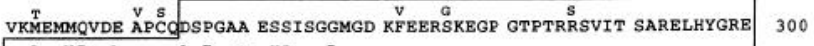

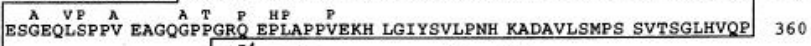
ALAVSMDFST YGGLLPOGFI QRELFSKLGE LAVGMKAESR PLGEQCSVCG VELPDNEAVE 420 QHRKLHSGMK TYGGELCGKR FLDSLRLRMH LLAHSAGAKA FVCDQCGAQF SKEDALETHR 480 QTHTGTDMAV FCLLCGKRFQ AQSALQQHME VHAGVRSYIC SECNRTFPSH TALKRHLRSH 540 TGDHPYECEF CGSCFRDEST LKSHKRIHTG EKPYECNGCG KKFSLKHQLE THYRVHTGEK 600 PFECKLCHQR SRDYSAMIKH LRTHNGASPY QCTICTEYCP SLSSMQKHMK GHKPEEIPPD 660 WRIERTYLYL CYV*

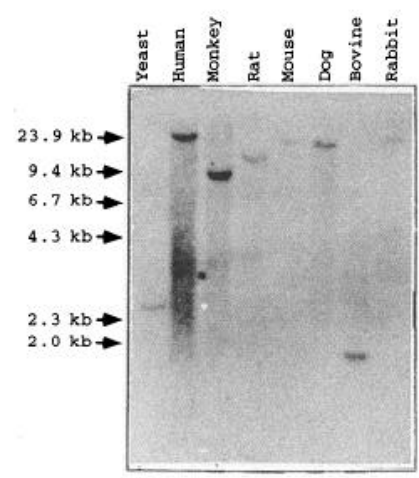

Figure 1. A schematic representation of the murine $P L Z F$ protein and probes used, is shown in $a$. In $a$, the dotted triangles represent nine zinc finger motifs; the location of the alternative exon, the presence or absence of which gives rise to two distinct isoforms (in human), the position of the initiation codon, and the break point (bpt) identified in the translocation involving the retinoic acid receptor $\alpha$ gene, are also indicated. The corresponding location of three probes is shown under the cDNA. These are as follows: P6 is a human cDNA fragment used in the screening of a murine cDNA library; $A S$ and $P 5$ are the murine probes used, respectively, in the Southern Zoo blot experiment and in the in situ hybridization analysis (see Materials and Methods). $b$, is the putative full coding sequence of the murine $P L Z F$ gene. Its coding sequence was compared to the human PLZF, and divergent amino acids in the human sequence are written above their mouse counterparts. In $b$, the POZ domain at N-terminal is double underlined, the alternative exon is boxed, the nine zinc fingers are underlined, and the position of the primers utilized to produce the AS ( $\mathrm{m} 5$ and $\mathrm{m} 6$ ) and P5 ( 4 and $\mathrm{n} 5$ ) probes are also indicated. $c$ Represents the nucleotide and corresponding amino acid sequences of the P5 probes. Numbers indicate the corresponding amino acid position in the PLZF coding sequence. $d$ Shows the PLZF cross-hybridization to genomic DNA of several eukaryotic species. In $d$, each lane contains approximately $8 \mu \mathrm{g}$ of genomic DNA digested with the restriction enzyme EcoRI from the following species: lane 1, yeast; lane 2, human; lane 3 , monkey; lane 4 , rat; lane 5 , mouse; lane 6 , dog; lane 7 , bovine; lane 8 , rabbit. The $\lambda \mathrm{DNA} /$ HindIII size markers are indicated on the left.

the mouse $P L Z F$ cDNA by PCR: P5, a 510 bp fragment contained within the $P L Z F$ region encoding the zinc finger motifs; and AS, a 633 bp fragment from the PLZF central region. The positions of the P6, AS, and P5 DNA probes with respect to the $P L Z F$ protein are shown in Figure $1, a$ and $b$. Nucleotide and corresponding amino acid sequences of the mouse P5 DNA fragment is shown in Figure 1c.

The extent of $P L Z F$ conservation throughout evolution has been investigated by Southern blot analysis. DNA isolated from the following eukaryotic species: yeast, human, monkey, rat, mouse, dog, bovine, and rabbit were hybridized with the PLZF cDNA probe AS. The Southern blot filter was washed as described and exposed for 12, 96, and $168 \mathrm{hr}$ (see Materials and Methods). After the longest exposure, a single band of the expected size was detected in the lanes corresponding to human and mouse DNA, indicating a specific hybridization to $P L Z F$ sequences and not to $P L Z F$-related genes (Fig. $1 b$ ). In the same experiment cross-hybridization to the AS probe was detected in all the tested species (Fig. 1b). These findings suggest that PLZF is conserved throughout evolution from yeast to mammals.

\section{PLZF early expression}

The expression pattern of $P L Z F$ has been studied during mouse embryonic development between E 6.0 and E 17 performing in situ hybridization experiments on embryo sections. For these experiments, we used the cDNA probe P5 (Fig. 1c). Control experiments with the sense strand of the same probe show no detectable signals (data not shown).

The first detectable hybridization signal is present at E 7.5. A sagittal section at this stage (Fig. $2 a$ ) shows that PLZF transcripts appear only in the neuroectoderm of the presumptive head fold.

At $\mathrm{E} 8$, when the node is posteriorly regressed and the neuroectoderm is induced, $P L Z F$ is strongly activated along the neuroectoderm (Fig. $2 b$ ). At E 8.5 , besides the neuroectoderm, $P L Z F$ is transcribed in the allantois and in the definitive embryonic endoderm (Fig. 2c).

Anteriorly, $P L Z F$ is expressed in the endoderm filling all the foregut diverticulum, including the endodermal component of the heart pocket (Fig. 2c). Transverse sections confirm previous 

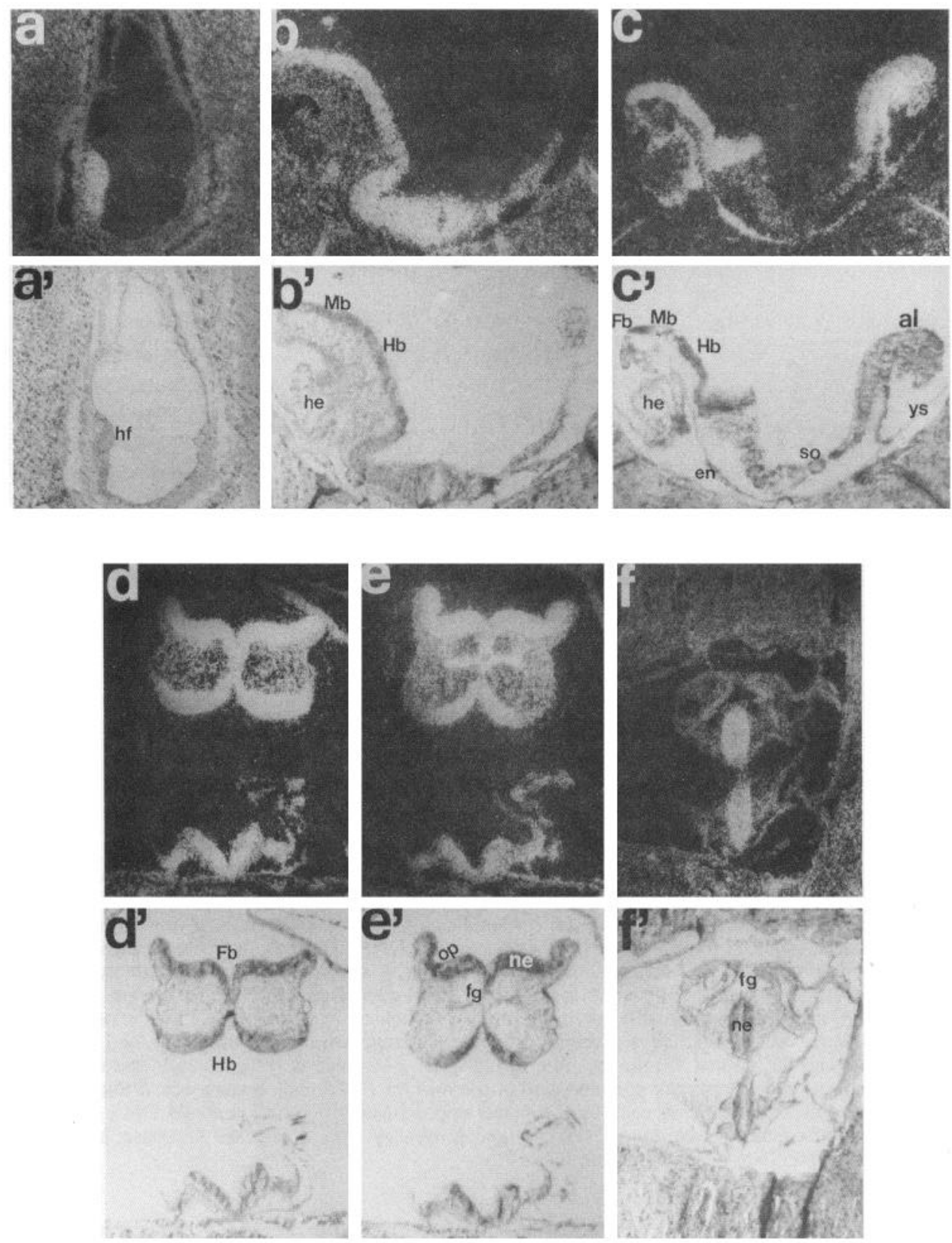

Figure 2. PLZF expression in sagittal sections of E $7.5(a)$, E 8-E $8.2(b)$, and $\mathrm{E} 8.5(c)$ mouse embryos. $d-f$ show the $P L Z F$ expression in coronal sections of E 8.5 mouse embryos. Bright fields of the same sections are indicated by a prime ('). Abbreviations: $F b$, forebrain; $M b$, midbrain; $H b$, hindbrain; $h f$, head fold; he, heart; $e n$, endoderm; so, somite; $a l$, allantois; $y s$, yolk sac; $n e$, neuroectoderm; $f g$, foregut; $o p$, optic pit. tle layer of the basal midbrain, as compared to the germinative layer (Fig. $3 g$ and data not shown) and in the region including the floor plate of the hindbrain (Fig. $3 j, k$ ). The clearest example of adjacent regions with different levels of $P L Z F$ transcripts can be seen by comparing the posterior to the anterior parencephalon (Fig. 3i,k).

Furthermore, the expression of $P L Z F$ also decreases in the regions of the tuberal and optic stalk of the hypothalamus (Fig. $3 g, i)$. Figure $4 a$ shows a detail of the $P L Z F$ expression in the spinal cord, indicating an ubiquitous distribution excluding only the basal mantle layer and the floor plate.

A gradual repression also appears in the mesenchyme of the branchial arches (Fig. 3h). It is worth briefly noting that this mesenchyme and that of the frontonasal prominence are derived from the cranial neural crest. Figure 6, $a$ and $d$ shows a detail of the optic cup and otic vesicle at E 10. In the optic cup, PLZF is expressed in the presumptive retina, but not in the lens vesicle. brain, $P L Z F$ transcripts are faintly detected in the primitive man- 

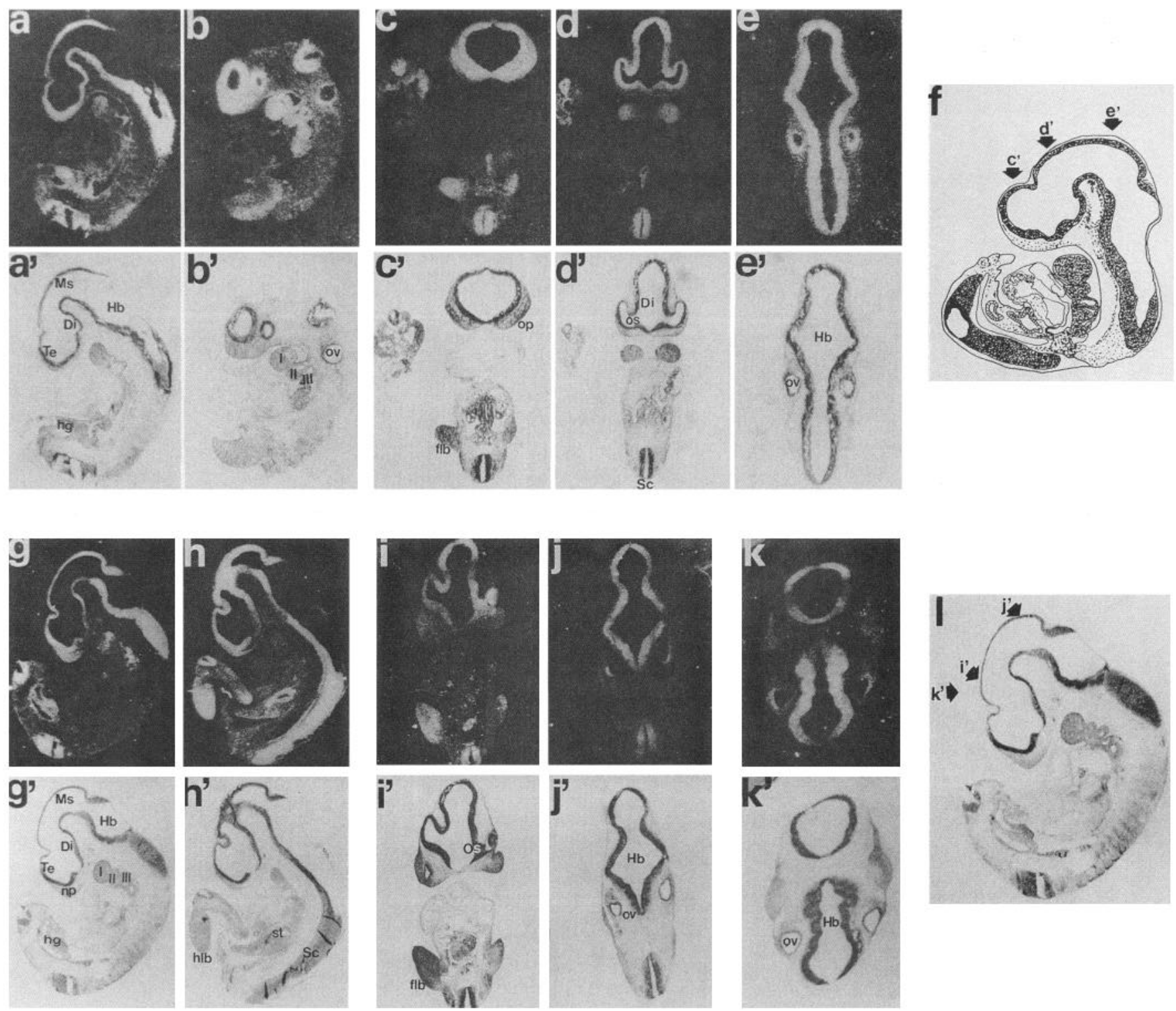

Figure 3. PLZF expression in sagittal $(a$ and $b)$ and frontal $(c-e)$ sections of E 9.5 mouse embryos and in sagittal $(g$ and $h)$, frontal $(i$ and $j)$ and coronal ( $k$ ) sections of E 10.5 mouse embryos. Bright fields of the same sections are indicated by a prime ('). A scheme of the frontal and coronal sections is also shown $(f$ and $l$ ). Te, telencephalon; $D i$, diencephalon; $M s$, mesencephalon; $S c$, spinal cord; $o v$, otic vesicle; $o p$, olfactory placode; $n p$, nasal process; $f l b$, forelimb bud; $h l b$, hindlimb; os, optic stalk; $h g$, hindgut; st, stomach; $m x$, maxillary process; $I, I I$, $I I I$ are, respectively, the first, second, and third branchial arch. Other abbreviations are as in Figure 2.

In the otic vesicle it is expressed at different level, gradually increasing along the mediolateral diameter of the structure. The expression analysis at earlier stages, from E 7.5 to E 10.5 , is suggestive of a role of $P L Z F$ in the early differentiative processes regarding either specific structures, (e.g., branchial arches components, visual, and acoustic sense organs) or early cell types (e.g., early neuroectodermal cells, early embryonic endoderm).

\section{PLZF expression at E 12.5}

We studied the PLZF expression pattern at E 12.5 in sagittal, frontal, and transverse sections. In sagittal sections, PLZF is detectable along the longitudinal body axis in the hindbrain and spinal cord (Fig. 5c).

In the hindbrain, PLZF is expressed in the germinal layer of the alar and basal plate with a remarkable increase at the level of the sulcus limitans (Fig. $5 d, e$ ), and in the primitive cerebellum (Fig. $5 c, j$ ); in the spinal cord it is transcribed both in the basal and alar plate (Fig. $4 b$ ). In the alar plate the hybridization signal seems to define a ventral border roughly corresponding to the sulcus limitans (Fig. 4b).

In the mesencephalon, PLZF is only expressed in the alarmost region including the roof plate (Fig. $5 c, j$ ), and in the forebrain it is regionally restricted, disappearing from many of the regions in which it was expressed at E 9.5 and E 10.5. Sagittal, frontal, and transverse sections through the diencephalon indicate that $P L Z F$ is expressed in the epithalamus, in the alarmost part of the dorsal thalamus (dorsal area) (Fig. $5 a-d, h$ ), and in a large stripe corresponding to the anterior pretectal area (Fig. 5a,i). This domain originates from the epiphyseal region located in the 


\section{E10}

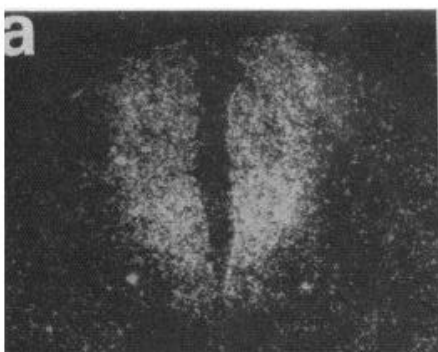

a'

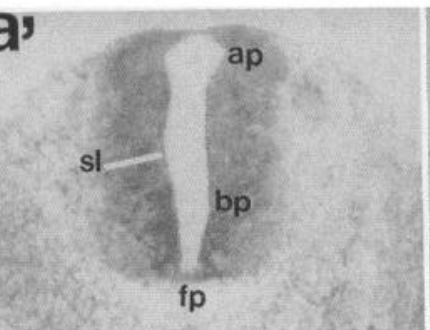

E12.5

E13.5
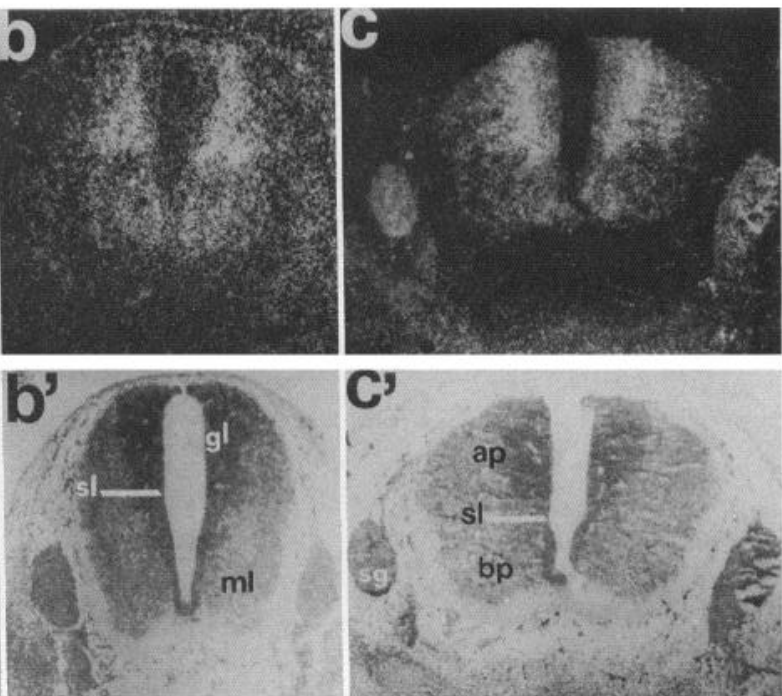

E14.5

Figure 4. PLZF expression in the spinal cord from E 10 to E 16.5 embryos $(a-e)$. The solid lines $\left(a^{\prime}-e^{\prime}\right)$ correspond to the presumptive sulcus limitans $(s l)$ and define the presumptive distinction of the alar (ap) from the basal plate $(b p)$. Abbreviations: $f p$, floor plate; $g l$, germinative layer; $m l$, mantle layer; $s g$, somatic ganglia; $d h$, $l h$, and $v h$ are, respectively, the dorsal, lateral, and ventral gray horn.
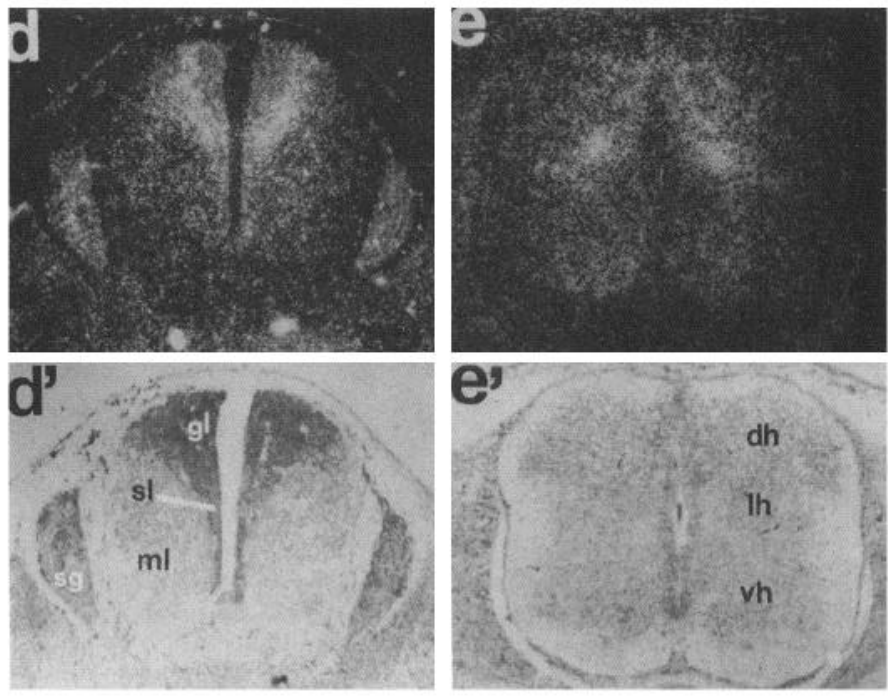

roof of the epithalamus and is closely parallel to the fasciculus retroflexus (the future habenulo-interpeduncular tract).

In the posterior pretectal region, including the posterior commissure, $P L Z F$ is only faintly expressed (see Figs. $5 a, i ; 9 d$ ). These results indicate that at $\mathrm{E}$ 12.5 PLZF is expressed in a segment-like pattern in the anterior pretectal region. This area corresponds to the neuromere D3 according to Figdor and Stern (1993). However, its expression is also found in the dorsalmost region belonging to the neuromere D2 (prosomere 2). In the hypothalamus, $P L Z F$ is detected in two nonoverlapping regions. The former includes the eminentia thalami, the supraoptic paraventricular region, and the preoptic area (Fig. 5a); the latter, the mammillary pocket (Fig. $5 b, c$ ). Transverse sections confirm these localizations (Fig. $5 d-f$ ).

In the telencephalon, $P L Z F$ is expressed in the septal area (Fig. $5 g$ ). Additional sites of expression are shown in detail in
Figure 6. $P L Z F$ is abundantly expressed in the sensory layer of the retina (Fig. $6 b$ ) and in the basal epithelium of the developing inner ear (Fig. 6e). Later on in development, the otic expression becomes undetectable (data not shown). PLZF is also detected in the infundibular recess (Fig. $5 f$; for a detail, see Fig. $6 f$ ) where the pars neuralis (posterior lobe) of the hypophysis is developing; in the mucosal layer of the stomach (Fig. 5i); in the duodenum; in the midgut loops (Fig. $5 c, h, i$ ) and, at lower levels, in the liver (Fig. $5 a, h-j$ ), in the intrinsic muscles of the tongue, in the lower jaw, possibly including the Meckel's cartilage (Fig. $5 b$ ) and in the prevertebrae (Fig. 5c).

\section{PLZF expression between $E 13.5$ and E 15.5}

The overall expression pattern of $P L Z F$ persists unaltered from that seen at E 12.5, also at E 13.5, E 14.5, and E 15 (data not shown). Nevertheless, several differences appear. At E 13.5, 

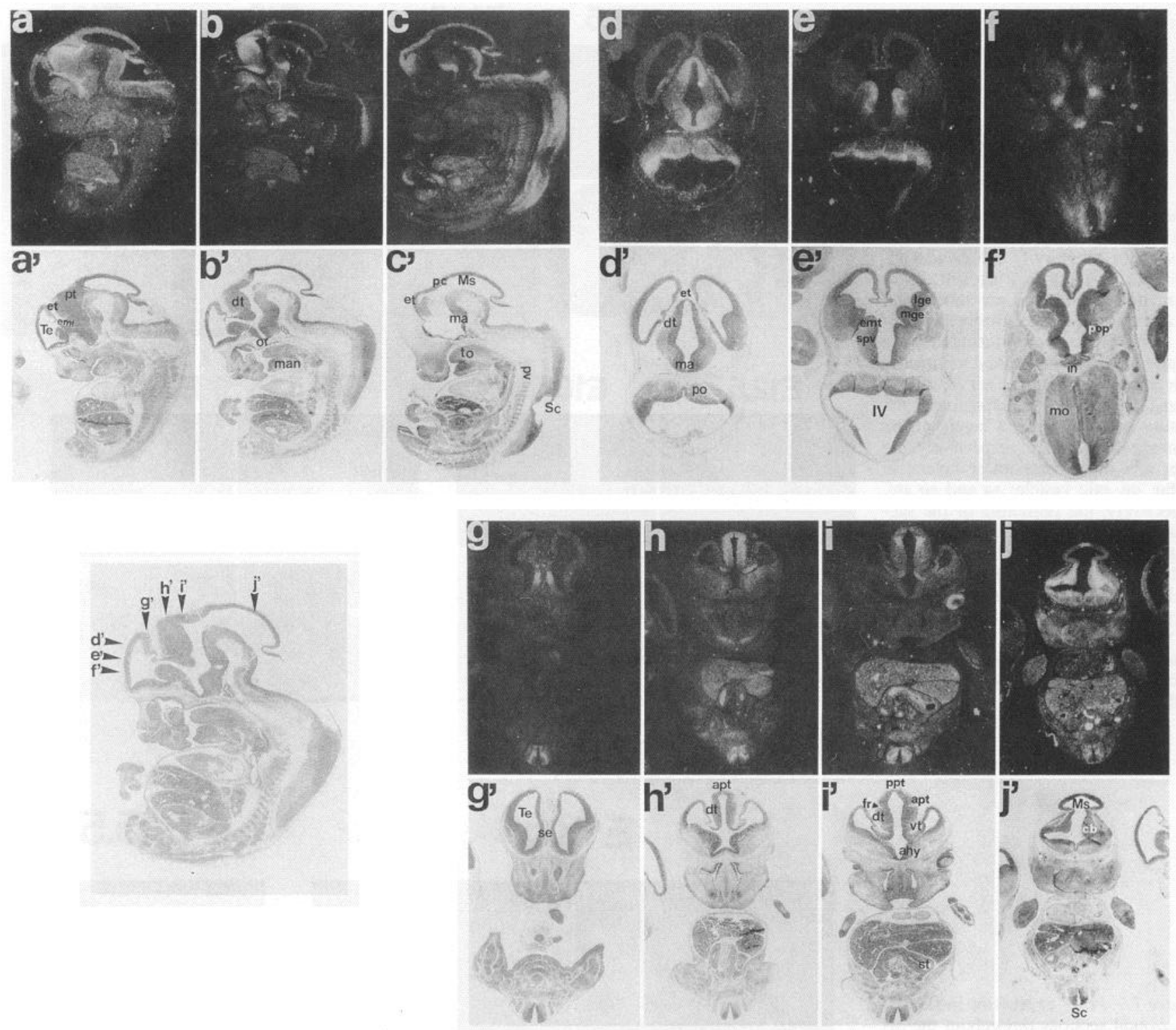

Figure 5. PLZF expression in sagittal $(a-c)$, coronal $(d-f)$, and frontal $(g-j)$ sections of E 12.5 mouse embryos. Bright fields of the same sections are indicated by a prime ('). Schemes of the frontal and coronal sections are also shown. $p c$, posterior commissure; $p t$, pretectum; $o r$, optic recess; man, mandible; to, tongue; $p v$, prevertebrae; $p o$, pons; mo, medulla oblongata; ahy, anterior hypothalamus; $c b$, primordium of the cerebellum; $f r$, fasciculus retroflexus; $s e, e t, d t, v t, o p t, p p t$, emt, $m a, s p v, p o p, m g e$, lge are listed in the appendix. Other abbreviations are as in previous figures.

$P L Z F$ is still expressed in the hindbrain and along the spinal cord.

In the spinal cord, $P L Z F$ is detected in the external germinal layer of the alar plate (Fig. $4 c$ ) and at E 14.5 is clearly restricted to the same area, even if a weak signal persists also at the level of the internal germinal layer both of the alar and basal plate (Fig. 4d).

In the diencephalon, $P L Z F$ is detected in the epithalamus, in the dorsal thalamus, in the anterior and posterior pretectum, and in restricted hypothalamic areas (Fig. 7a-c) including, at E 13.5, the basal tuberal area (Fig. $7 b$ ). Furthermore, a new suggestive expression domain is represented by a thin stripe morphologically coincident with the zona limitans intrathalamica (Fig. $7 a, c)$. To carefully assess this point we hybridized frontal adjacent sections to $P L Z F$ and $D l x l$ genes. Dlxl has been exten- sively investigated because of its interesting segment-like expression pattern in the ventral thalamus (Price et al., 1991; Bulfone et al., 1993a; Simeone et al., 1994a,b).

By comparing $P L Z F$ and $D l x l$ (Fig. $7 c, d$ ), we found that the $P L Z F$ thin stripe is exactly adjacent to the border of $D l x l$, thus unambiguously confirming its location at the level of the zona limitans intrathalamica. This boundary distinguishes the dorsal thalamus from the ventral thalamus, and represents the boundary between the posterior and the anterior parencephalon, between the neuromeres D1 and D2 (Figdor and Stern, 1993), and the prosomeres $\mathrm{p} 2$ and $\mathrm{p} 3$ (Puelles and Rubenstein, 1993). At E 15.5 the zona limitans intrathalamica corresponds to the lamina medullaris externa where PLZF is detected (Fig. 7e). At E 15.5, $P L Z F$ transcripts are still restricted in a segment-like domain to the anterior pretectal area (Fig. 7e). 
Figure 6. $\quad P L Z F$ expression in visual $(a-c)$ and acoustic ( $d$ and $e$ ) sense organs, in the pituitary ( $f$ and $g$ ), and adrenal ( $h$ and $i$ ) glands. Stages of developing mouse embryos are labeled on the top of each image. Bright fields of the same sections are indicated by a prime ('). Abbreviations: $o c$, optic cup; $l v$, lens vesicle; on, optic nerve; $l f$, lens fibers; $s l r$ and $p l r$ are, respectively, the sensory and the pigment layers of the retina; inl and onl are, respectively, the inner and the outer nuclear layers; $e$, eyelid; $o v$, otic vesicle; $s a$ and $c o$ are, respectively, the primordia of the sacculus and cochlea; VIII is the acoustic ganglion, inr, infundibular recess; $R p$, Rathke's pocket; $a l$ and $p l$ are, respectively, the anterior and posterior lobe of the pituitary gland; $p i$, pars intermedia of the pituitary gland; $a g$, adrenal gland; $k$, kidney; $c o$ and $m e$ are, respectively, the cortex and the medulla of the adrenal gland.

Figure 7. $\quad P L Z F$ expression in sagittal and frontal sections throughout the diencephalon of E $13.5(a-c)$ and E 15.5 (e) mouse embryos. At E 13.5, PLZF expression $(c)$ is compared to an adjacent frontal section hybridized with $D l x l$ gene $(d)$. Bright fields of the same sections are indicated by a prime ('). hi, hippocampal primordium; zli, zona limitans intrathalamica; eml, lamina medullaris externa; $e t, d t, v t, a p t$, $p p t$, in, $m a$, and $t u$ are listed in the appendix. The open arrow in $a^{\prime}$ indicates the level at which the frontal section $\left(d^{\prime}\right)$ was made. The arrows in $a$ and $a^{\prime}$ point, respectively, to the $P L Z F$ hybridization and to the histology in the presumptive zona limitans intrathalamica. The same $P L Z F$ hybridization in the zona limitans intrathalamica and corresponding histology is also marked by two arrowheads, respectively, in $c$ and $d^{\prime}$. Note that the $P L Z F$ hybridization signal is adjacent to the $D l x l$ expression domain in the ventral thalamus (d). The arrows in $e$ and $e^{\prime}$ point to the $P L Z F$ expression in the lamina medullaris externa. Other abbreviations are as in previous figures.
E10
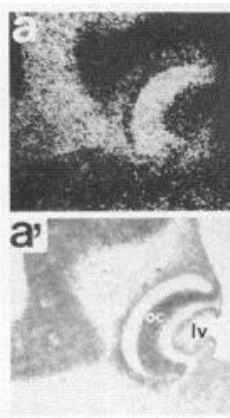

E12.5
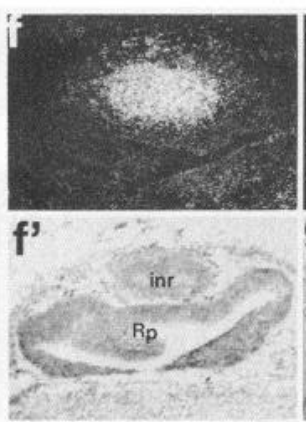

E12.5
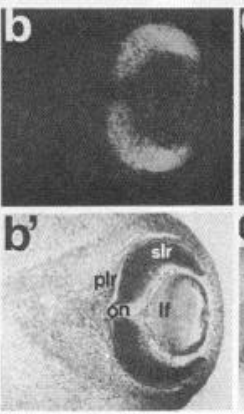

E16.5

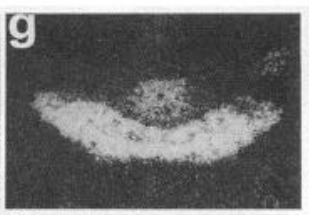

g'

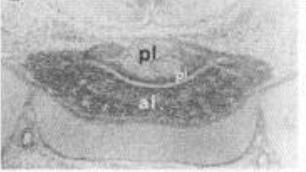

E10
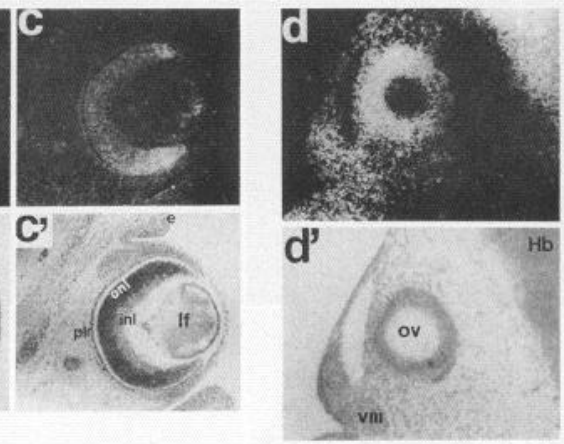

\section{E13.5}
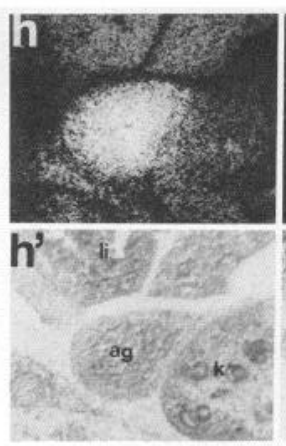

e'

E12.5
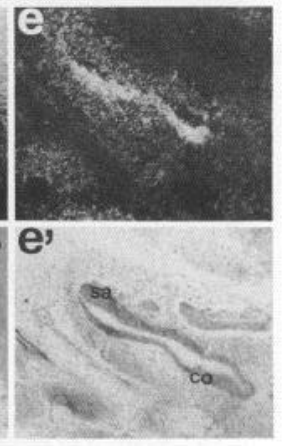

E16.5

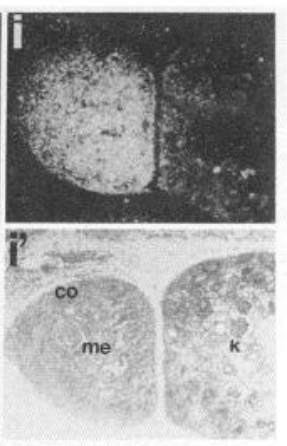

\section{E13.5}
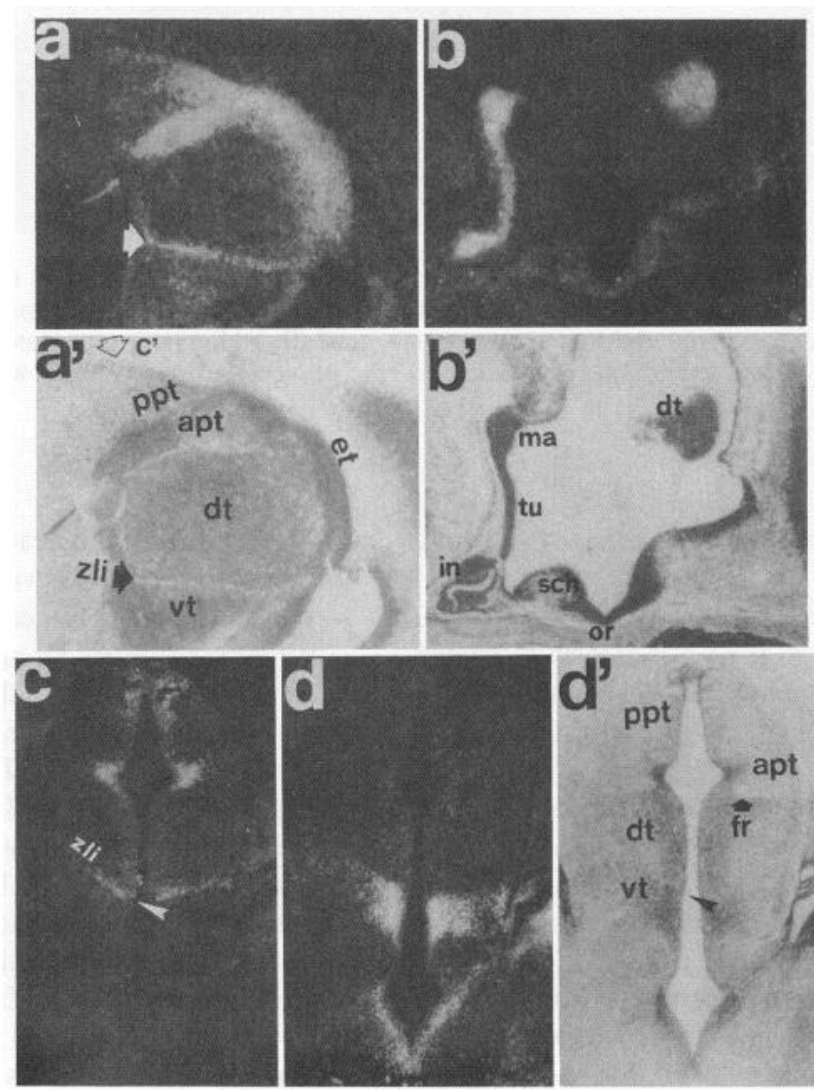
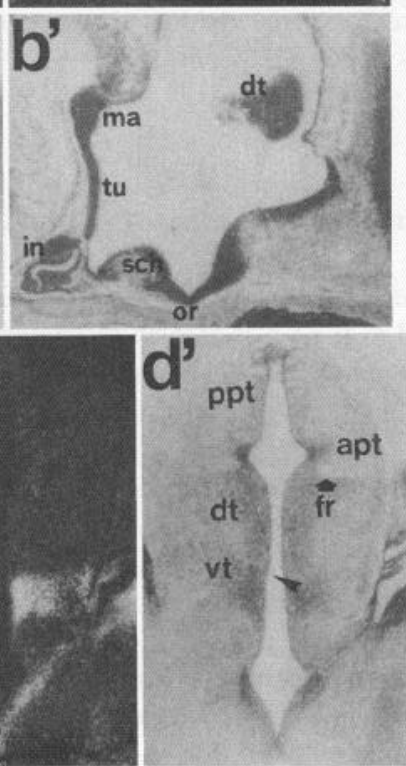

E15.5
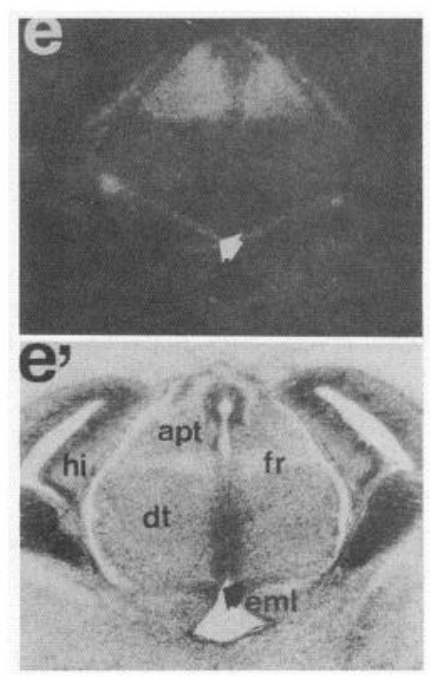
In summary, these new findings support a role for $P L Z F$ in the establishment and/or maintenance both of regional identities and segmentary boundaries.

$\Lambda$ dditional sites of expression at E13.5 include the stomach, where the expression persists with a higher level in the mucosal layer (data not shown), the infundibular recess (Fig. $7 b$ ), the developing thymus (data not shown), and the adrenal gland (Fig. $6 h)$.

Finally, at E15.5, PLZF is detected in the retina in close proximity of the future ciliary body (Fig. 6c) and at higher levels in the liver (data not shown).

\section{Late expression at E 16.5}

$P L Z F$ expression in the spinal cord is most abundant in the dorsal-alar plate and in a restricted area of the intermedial region (Fig. 4e). We assigned the alar expression to a subregion of the somatic-sensorial area and the intermedial signal, detected as two large spots, to the visceral-sensorial area of the lateral gray horn (Fig. 4e). In the hindbrain, PLZF is faintly expressed both in the basal and alar plate (data not shown), and in the rostral CNS it is abundantly transcribed in a restricted area of the dorsal thalamus (Fig. 8a,c) and in the whole anterior pretectal area (Fig. $8 a, b, d)$. However, PLZF is detected also in the epithalamus, ventral thalamus, hypothalamus, and hippocampal cortical layer (Fig. $8 c, d$ ). We have attempted to assign the $P L Z F$ expression domains to specific nuclei and regions. We suggest that the PLZF expressing regions include the ventrolateral nucleus (Fig. $8 c$ ) in the dorsal thalamus; the medial habenula in the epithalamus (Fig. 8c); the paraventricular and the periventricular nuclei (Fig. $8 c, d$ ) in the hypothalamus; several small spotted regions in the zona incerta and in the reticular nucleus (Fig. $8 d$ ) of the ventral thalamus; the whole anterior pretectal area including related nuclei (Fig. 8a,b); the presumptive lamina medullaris externa (Fig. 8d). Additional structures expressing $P L Z F$ include the thymus (data not shown) and the anterior and posterior lobes of the pituitary gland (Fig. $6 \mathrm{~g}$ ). It is worth noting that at E 16.5 the endocrine organs such as the anterior and posterior lobes of the pituitary gland, the thyroid (data not shown), and the adrenal gland (Fig. 6i) actively transcribe $P L Z F$, suggesting that this gene might represent a common relevant factor during the development of these organs.

\section{Comparison between PLZF, Otx1, Otp, and Dlx1 expression domains}

The results so far described indicate that $P L Z F$ shows a very dynamic pattern during neurogenesis. In the forebrain, $P L Z F$ expression is regionally restricted to derivatives of the posterior parencephalon, synencephalon, and secondary prosencephalon, and defines neuromeric boundaries (Figdor and Stern, 1993; Puelles and Rubenstein, 1993), suggesting its involvement in the establishment and/or differentiation of segmental identities. Therefore, to confirm this evidence, we have tried to define exactly the spatial relationships between $P L Z F$ and other regulatory genes segmentally expressed in the forebrain. In fact, examples of related expression patterns between homeobox-containing genes (e.g., Hoxb- 1 ) and zinc finger genes (e.g., Krox20) have already been suggested (Wilkinson et al., 1989a,b; Marshall et al., 1992). Moreover, other gene families, vertebrate homologs of Drosophila regulatory genes (Jessell and Melton, 1992; McMahon et al., 1992; Smith, 1994), seem to control the identity and the correct development of specific regions of the CNS. Although in mammals there is no direct evidence for a functional link between different families of regulatory genes, their expression patterns strongly suggest the existence of regulatory interactions. Members of the zinc finger family could play a role in these interactions.

We compared the expression pattern of Otxl, Otp, Dlxl, Emxl, Emx2, and Otx2 with that of PLZF at E 12.5. The expression patterns of these genes have previously been documented (Simeone et al., 1992b, 1993, 1994a,b; Bulfone et al., 1993a; Puelles and Rubenstein, 1993). We only show more significant spatial relationships among $P L Z F$ and OtxI, Otp, and $D l x 1$ in sagittal, transverse, and frontal serial sections (Fig. 9).

Besides the expression in the germinal layer of the dorsal thalamus and epithalamus, which are not evident in Figure $9 a$, the Otxl expression pattern appears particularly interesting in the pretectal area (Fig. 9a) where its strongest signal appears adjacent to the $P L Z F$ domain in the anterior pretectal area and restricted to the posterior pretectal area (Fig. $9 d$ ).

In the hypothalamus, an additional morphological relationship between $O t x I$ and $P L Z F$ is represented by the narrow stripe running between the mammillary region, the ventral thalamus, the hypothalamic cell cord, the entopeduncular area, and the dorsal tuberal area (Fig. $9 a, d, d^{\prime}$ ). Also, Otp is expressed exactly in the same area (Fig. 9, compare $b$ and $d$ ). Interestingly, it appears that this narrow stripe expressing Otp and PLZF defines the posterior/ventral border of $D l x l$ (Fig. 9c). Furthermore, Otp and $P L Z F$ are coexpressed in a longitudinal area entirely included in the hypothalamus and subdivided in transverse neuromeric regions corresponding to the supraoptic/paraventricular area, the anterior hypothalamic area, and the preoptic posterior area (Fig. 9b,d,d') (Bulfone et al., 1993a). In this region, PLZF and $O t p$ are complementary to $D l x l$ (Fig. 9c) (Simeone et al., 1994b). The complementarity between $P L Z F$ and $D l x I$ is emphasized by the additional $P L Z F$ expression in the eminentia thalami (Fig. $9 c, d, d^{\prime}$ ). Transverse serial sections through the hypothalamus, along the plane indicated in Figure $9 d^{\prime}$, confirm the relationships deduced from sagittal sections, between $P L Z F$, Otp, and Dlxl. PLZF is expressed at this level in the anterior hypothalamic area (Fig. 9h). By comparing the PLZF expression pattern to that of Otp (Fig. 9f), it appears that the former is detected in two single spots included in the latter. Both $P L Z F$ and Otp are complementary to $D l x l$ (Fig. $9 g$ ). Despite the complementary pattern observed in the hypothalamus, in adjacent frontal sections through the plane indicated in Figure $9 d^{\prime}$, we found that $P L Z F$ is expressed in a region included in the $D l x I$ domain (Fig. 9k,l). This area corresponds to the external germinal layer of a restricted subregion of the ventral thalamus. It is worth noting that the $P L Z F$ localization in the external germinal layer of the ventral thalamus is also complementary to that of $O t x 1$, which is expressed in the internal germinal layer (Fig. 9i).

\section{PLZF regulation by retinoic acid $(R A)$}

Since $P L Z F$ is expressed early during mouse development and its later expression indicates a possible role in the establishment of CNS identities, we studied the effect of RA on the PLZF expression pattern both in vivo, by administering RA to early pregnant mice, and in vitro, in a human teratocarcinoma cell line (NT2/clone D1). Precise effects of RA both in vivo and in vitro have been extensively described (Simeone et al., 1990, 1991; Conlon and Rossant, 1992; Marshall et al., 1992; Simeone, Avantaggiato, Moroni, Mavilio, Arra, Cotelli, Nigro, and Acampora, unpublished observations). In vivo RA administration at 

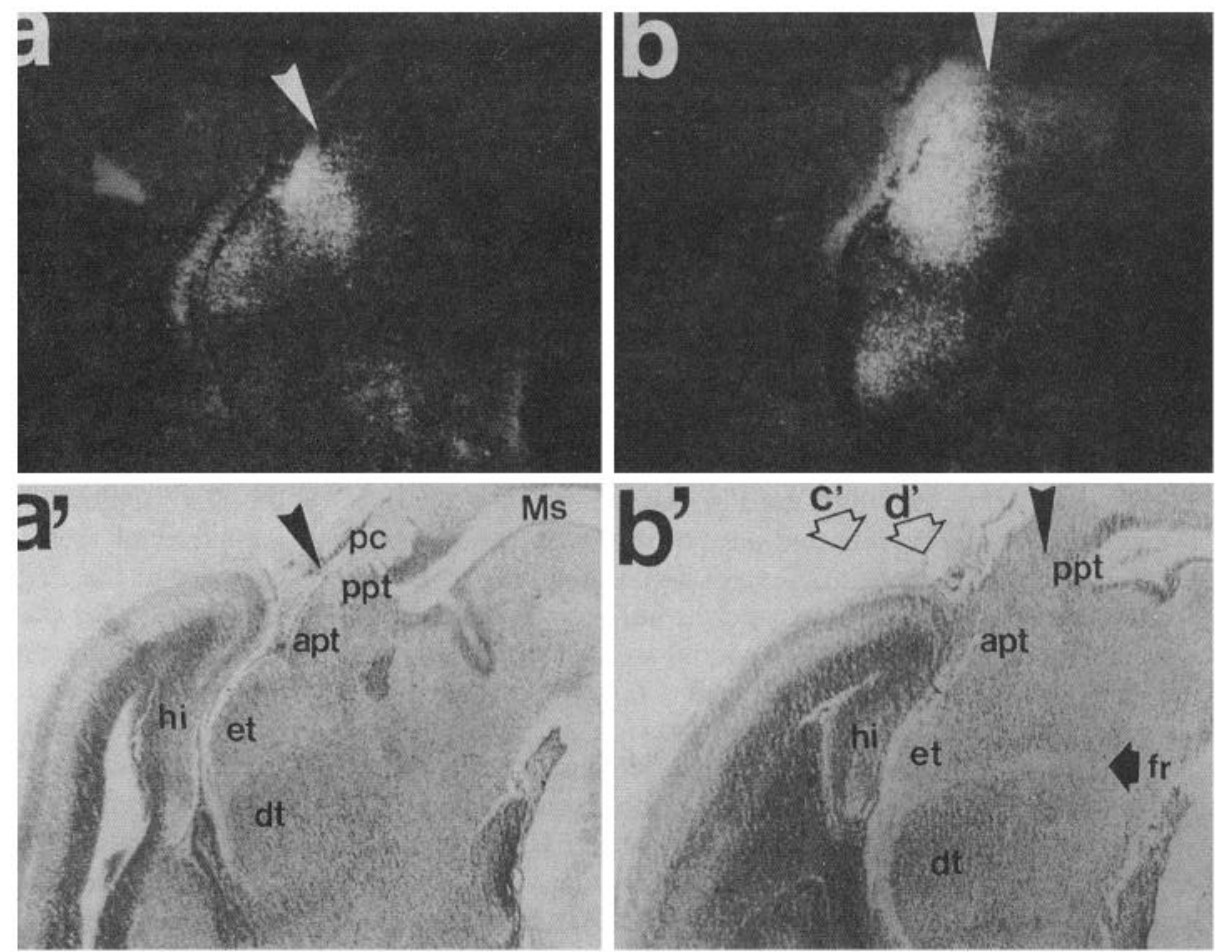

Figure 8. $\quad P L Z F$ expression in sagittal $(a$ and $b$ ) and frontal ( $c$ and $d$ ) sections of E 16.5 mouse embryos. Bright fields of the same sections are indicated by a prime ('). ham, medial habenula; pi, pineal recess; $v l$, ventrolateral nucleus; pan, paraventricular nucleus; pen, periventricular nucleus; $e t, d t, v t, a p t$, and ppt are listed in the appendix. Open arrows in $b^{\prime}$ indicate the levels at which the frontal sections $\left(c^{\prime}\right.$ and $\left.d^{\prime}\right)$ were made. Arrowheads in $a$ and $b$ and in $a^{\prime}$ and $b^{\prime}$ point, respectively, to the posterior border of the $P L Z F$ expression in the anterior pretectum and to the related histology. The filled arrow in $b^{\prime}$ points to the fasciculus retroflexus and in $d$ to the presumptive lamina medullaris externa. Other abbreviations are as in previous figures.
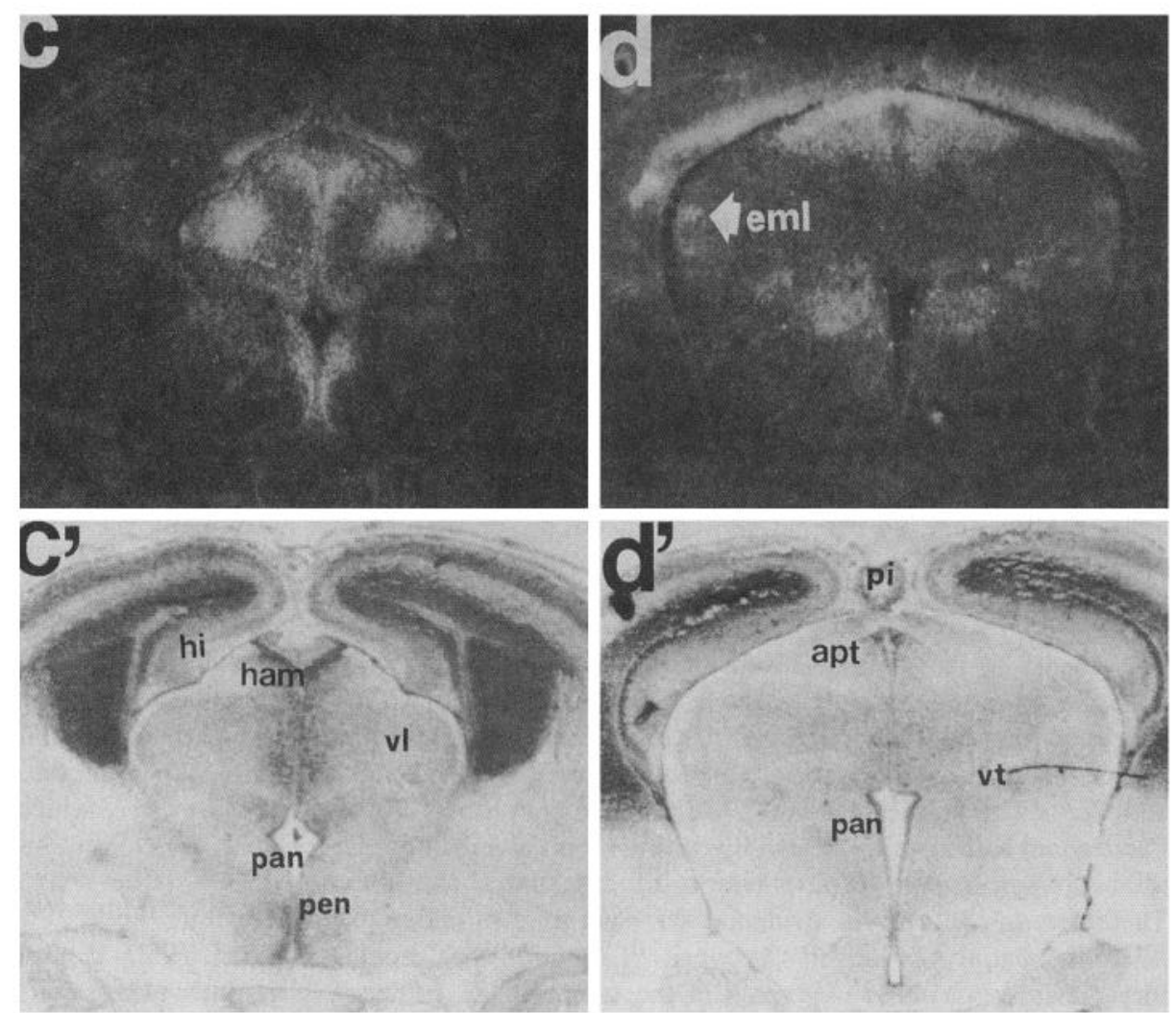

specific developmental stages results in stage-specific alterations. Embryos analyzed at E10.5 previously RA treated at E 7.8-E 8 (0-3 somite stage) show a remarkable neuroepithelial hyperproliferation (Fig. $10 c^{\prime}, d^{\prime}$ ) (Simeone, Avantaggiato, Moroni, Mavilio, Arra, Cotelli, Nigro, and Acampora, unpublished observations), on the other hand, E10.5 embryos RA treated at
E 7.2-E 7.4 (mid to late streak stage) show very severe alterations, including a strong hypoproliferative reduction of the neuroepithelium (Fig. 10 $a^{\prime}, b^{\prime}$ ) (Simeone, Avantaggiato, Moroni, Mavilio, Arra, Cotelli, Nigro, and Acampora, unpublished observations). Many of the RA-altered structures do express $P L Z F$ in untreated embryos. For these reasons, we hybridized $P L Z F$ 
Otx1
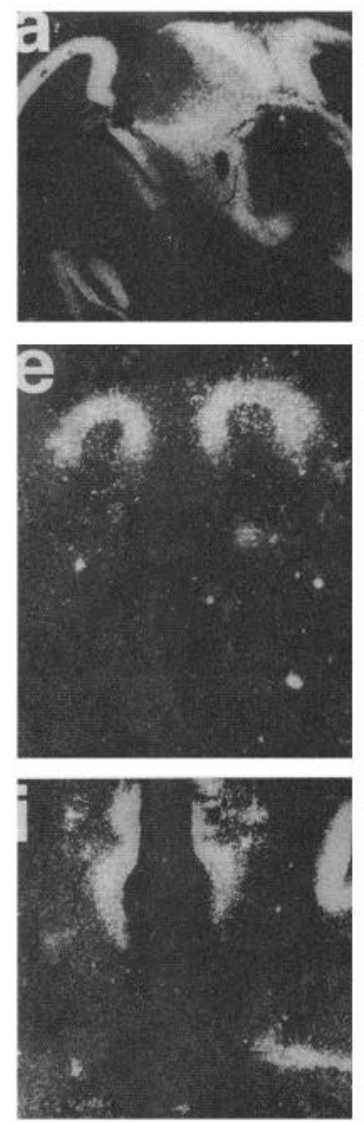

Otp
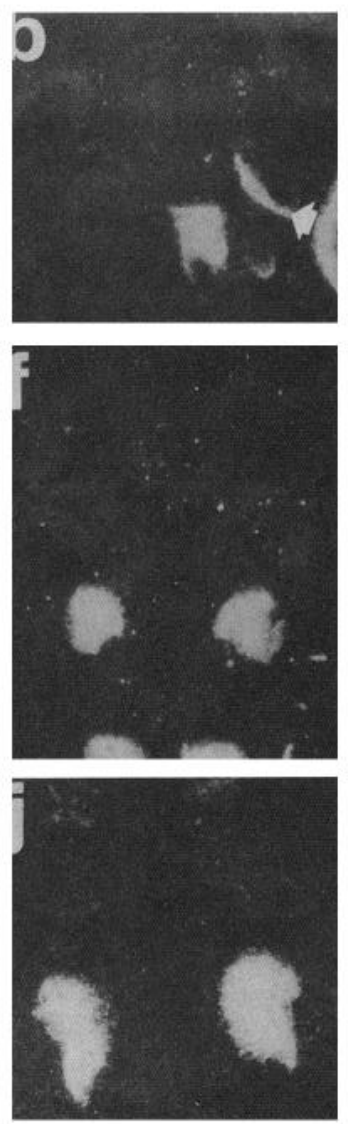

Dlx1
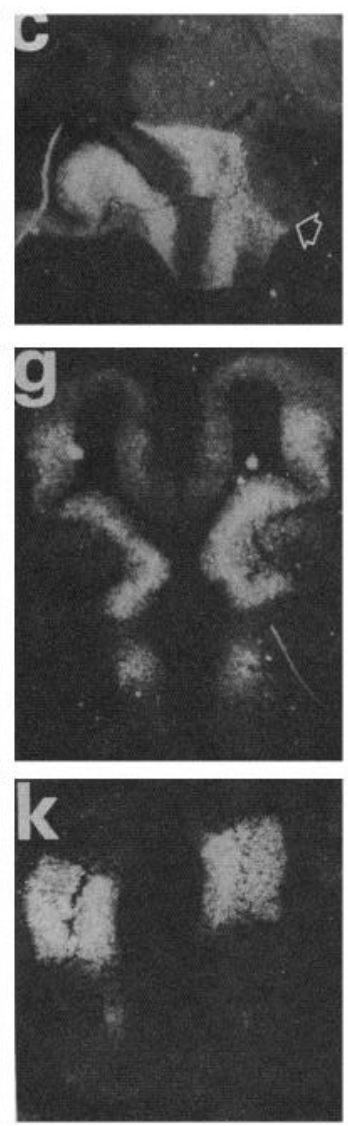

PLZF
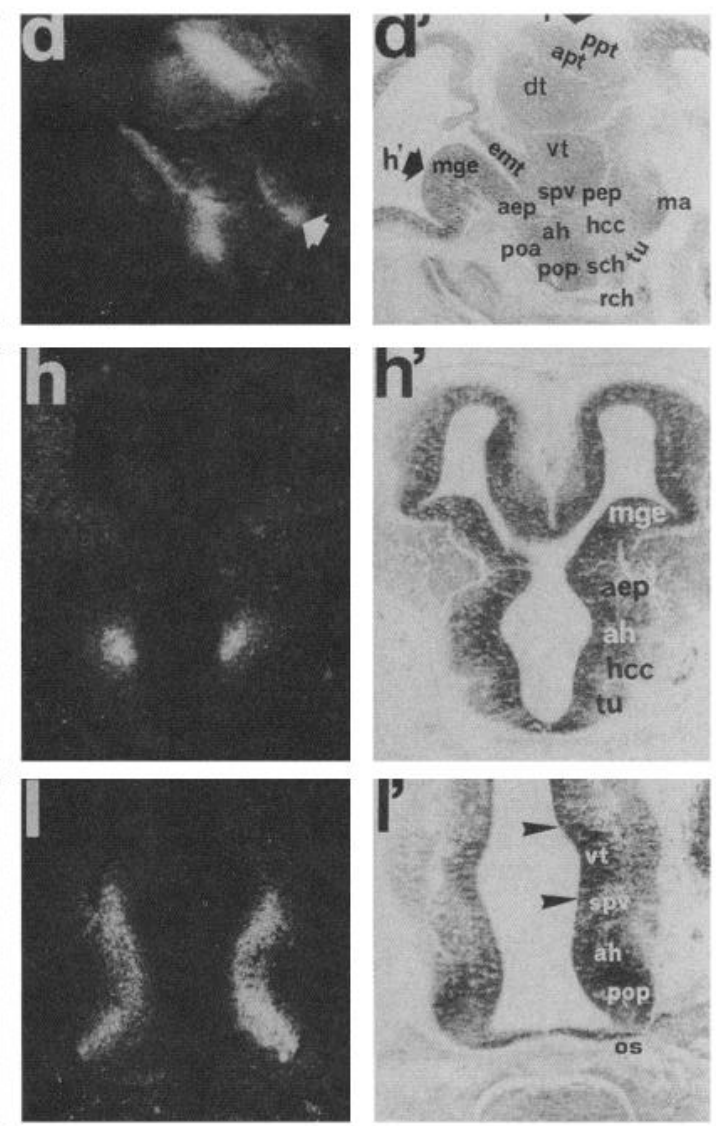

Figure 9. Detailed comparison of $O t x l$, Otp, Dlxl, and PLZF expression domains in sagittal ( $a-d)$ coronal $(e-h)$, and frontal $(i-l)$ sections in the diencephalon of E 12.5 mouse embryos. Hybridization was with Otxl $(a, e$, and $i), \operatorname{Otp}(b, f$, and $j), D l x I(c, g$, and $k)$ and $P L Z F(d, h$, and $l)$. Bright fields of the same sections are indicated by a prime ('). All the abbreviations are listed in the appendix. The filled arrows in $d^{\prime}$ indicate the level at which the coronal $\left(h^{\prime}\right)$ and the frontal $\left(l^{\prime}\right)$ sections were made. The filled arrows in $b$ and $d$ point to the PLZF and Otp common expression in a thin stripe bordering the posteroventral domain of Dlxl in the position indicated by an open arrow in $c$. The two arrowheads in $l^{\prime}$ define the $D l x l$ domain corresponding to the ventral thalamus.

to E 10.5 embryos treated with RA at E 7.4 and E 7.8 (Fig. 10). Our results show that $P L Z F$ is abundantly expressed in both types of RA-induced phenotypes, either in the neuroepithelium and sense organs, or in the limbs and branchial arches (Fig. 10ad) without qualitative changes in its expression pattern. In the strongest phenotype, the reduced number of districts expressing $P L Z F$ is simply due to the absence or reduction of several structures (e.g., sense organs, branchial arches, limb buds, neuroectoderm) (Fig. 10a,b).

On the other hand, $P L Z F$ is essentially expressed at the same level both in uninduced and RA-induced teratocarcinoma cells. However, since RA treatment of NT2/D1 cells results in the differentiation of many cell types, we cannot rule out the possibility that $P L Z F$ transcription is selectively induced into a specific cell type and repressed in others, resulting in a constant mRNA level when compared to the uninduced cells (Fig. 10e).

These results, however, suggest that RA does not modulate $P L Z F$ expression per se both in the NT2/D1 cell line and in early embryonic development.

\section{Discussion}

PLZF expression during early CNS development

In a small fraction of acute promyelocyte leukemia (APL) patients, the retinoic acid receptor $\alpha$ gene $(\operatorname{RAR} \alpha)$ is fused to
PLZF (Chen et al., 1993), a new Kruppel-like zinc finger gene that is found to be highly conserved throughout evolution from yeast to mammals. To elucidate the role of $P L Z F$, we studied its expression pattern in developing mouse embryos. The first general observation is that $P L Z F$ is mainly expressed in the CNS, suggesting that its principal role might be played during neurogenesis rather than hemopoiesis. The second observation is that $P L Z F$ is expressed in a variegate, extremely dynamic pattern. Our findings indicate two different behaviors for PLZF during embryonic development. The first is apparent between $\mathrm{E}$

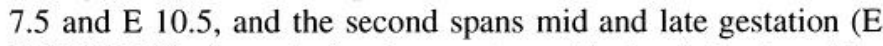
12.5-E 16.5). In early development, a critical role is played by specific morphogenetic signals operating when the neural pattern is established and controlling the expression of responding genes, which, in turn, interpret positional (dorso/ventral and antero/posterior) and differentiative signals (reviewed in Jessell and Melton, 1992; Ruiz i Altaba, 1993, 1994; Smith, 1994). When these signals are involved in establishing the neural pattern, PLZF is first expressed at E 7.5 in the head fold and is rapidly spread along all the neuroectoderm. Only a few hours later (5-8 somite stage) $P L Z F$ is detectable in many tissues of endodermal origin including those with inductive roles (e.g., 


\section{E7.4}
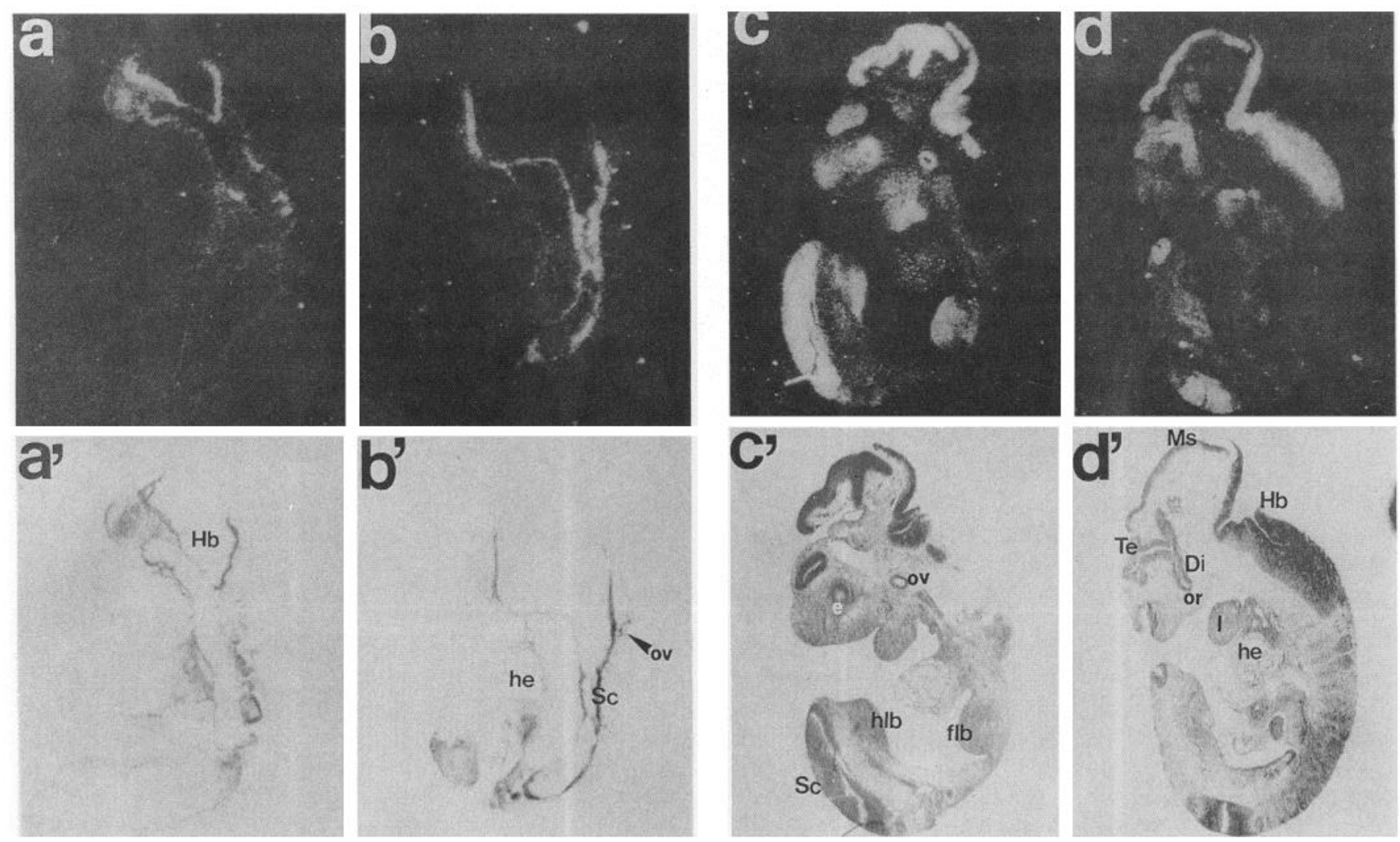

e

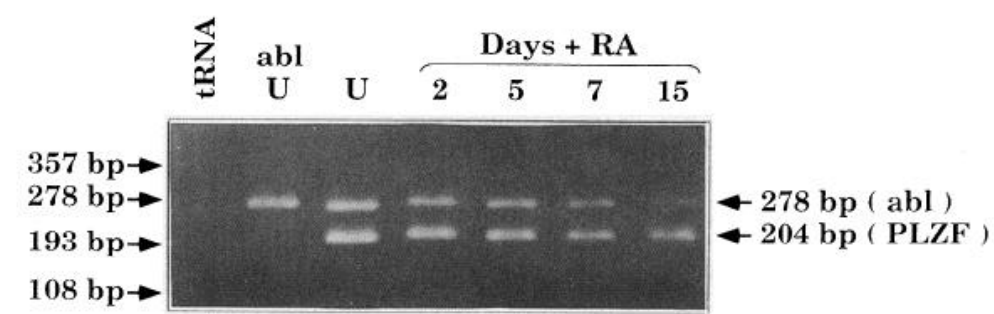

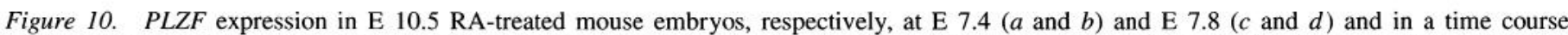

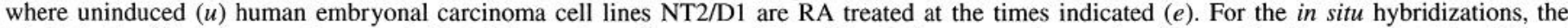

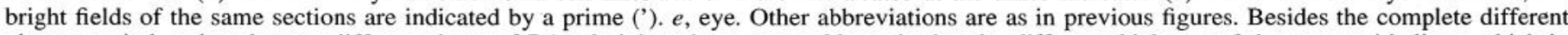

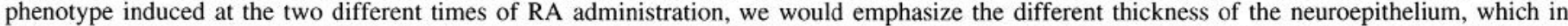

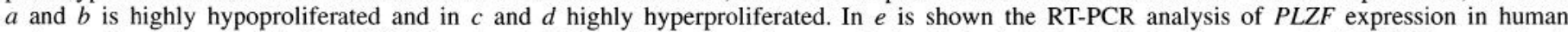

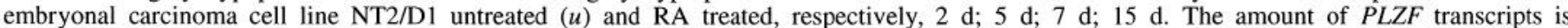

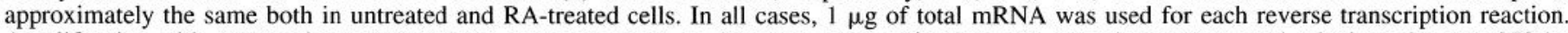

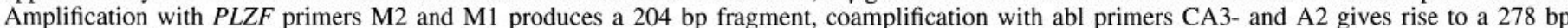

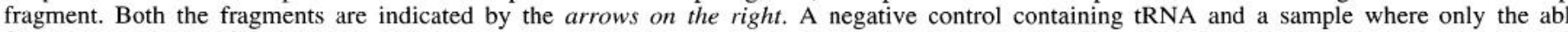
fragment can be amplified using NT2/DI RNA $(u$-abl) are also shown. On the left, pEMBL/TaqI/PvuII molecular weight markers are shown.

foregut endoderm). These findings indicate that, besides being an early endodermal marker, $P L Z F$ is mainly an early neural gene, gradually expressed from anterior to posterior according to the rostrocaudal neural pattern establishment. The ubiquitous distribution in the early neuroectoderm appears unrelated to the regional differentiation, but mainly to the primitive neuroectodermal cell type.

The ubiquitous presence of $P L Z F$ along the CNS is, however, a transient event. At E 10 PLZF begins to disappear from spe- cific regions of the CNS. This regional repression could be related to the morphogenetic events that take place around E 10 . These morphogenetic processes involve cellular differentiation and migration and represent the first event in the regional differentiation of the neuromeric identities. At this stage, several regionally restricted genes (e.g., the Dlx family, Orthopedia, the NK family, etc.) (Price et al., 1991, 1992; Bulfone et al., 1993a,b; Simeone et al., 1994a,b) begin to be activated in defined areas of the forebrain subventricular neuroepithelium. His- 
tochemical studies on rat and chick embryos indicate that the intricate pattern of nuclei formation in the diencephalon arises from the early differentiation of the neuroepithelium (Altman and Bayer, 1978, 1979; Puelles et al., 1987). At E 10-E 10.5, when the mentioned homeobox-containing genes are regionally activated, $P L Z F$ begins to be repressed and gradually confined to restricted areas. We attempt to speculate on this finding, suggesting that the early ubiquitous expression is a temporally regulated event with a possible negative effect on the early differentiation program of specific regional identities corresponding to those where PIZF disappears. Moreover, since the PI.ZF expression at $\mathrm{E} 12.5$ becomes restricted to defined neuromeric regions, we also suggest that $P L Z F$ represents a putative necessary factor in the correct development of posterior parencephalic and synencephalic derivatives as well as subregions of the hypothalamus. The gradual repression of a putative regulatory gene, such as $P L Z F$, opposed to the activation of other regulatory genes, opens up a new aspect in the complex morphogenetic program that gives rise to regional identities in the early commitment of the forebrain regions.

\section{PLZF expression in the CNS mid to late gestation: implications for the neuromeric model}

The anatomy of the rostral brain has been the focus of a wide number of morphological and molecular studies. Two main models have been postulated, named the columnar and, more recently, the neuromeric models. The columnar model (Herrick, 1933; Kuhlenbeck, 1973) proposes the existence of four longitudinal columns, the Herrick's columns, representing histogenic compartments separated by ventricular sulci such as the sulci diencephalic dorsalis, medius, and ventralis. The direction of these sulci defines the longitudinal axis. The neuromeric model (Puelles and Rubenstein, 1993; Rubenstein et al., 1994) postulates the existence of both longitudinal and transverse domains. The principal longitudinal domains are represented by the alar and basal neuroepithelium extending along all the CNS. The alar and basal longitudinal regions in the fore-, mid-, and hindbrain are subdivided into transverse domains (Bulfone et al., 1993a; Puelles and Rubenstein, 1993). Molecular analysis of many regionally restricted genes appears more consistent with the neuromeric model (reviewed Puelles and Rubenstein, 1993). Furthermore, the expression patterns of these genes suggest that the alar domain of the secondary prosencephalon can be subdivided in secondary longitudinal subdomains.

On the other hand, previous results showing a general subdivision into four large neuromeres, each of them representing a discrete unit of a polyclonal cell population with restricted lineage (Figdor and Stern, 1993), are consistent with the segmental nature of the diencephalon. In this case as well, the expression domains of several regulatory conserved genes fill zones or structures defined by polyclonal cell lineage restrictions and morphology (Figdor and Stern, 1993).

Neuromeric and subneuromeric identities are distinguished by defined boundaries. Their existence is frequently morphologically revealed but, in several cases, suggested on the basis of the expression domains of segmentally restricted genes. This is particularly true in the case of subneuromeric boundaries.

We show that $P L Z F$ is expressed in a complex pattern of expression in the developing forebrain and that its pattern is suggestive of a role in the definition and establishment of transverse (neuromeric), longitudinal domains, and intraneuromeric boundaries. In order to clarify these findings, we report the $P L Z F$ expression domains in a schematic representation of the longitudinal and transverse domains of the rostral CNS. To this end we have used the scheme proposed by Puelles and Rubenstein (1993). The $P L Z F$ expression pattern was compared to those of other segmentally restricted genes such as Otp and Dlxl (Fig. 11) (Price et al., 1991; Bulfone et al., 1993a; Simeone et al., 1994a,b).

These genes have been particularly useful in our analysis because of their expression in domains adjacent to or overlapping those of $P L Z F$ (Fig. 11).

From this analysis it is clear that $P L Z F$ expression identifies an extended longitudinal subdomain including the hypothalamic region where Otp is expressed, a subregion of the ventral thalamus, the alarmost dorsal thalamus, and the epithalamus. Despite the predicted longitudinal domain within the hypothalamus, no evidence exists of additional longitudinal subdivision of the ventral and dorsal thalamus even if a similar behavior with a decreasing dorsoventral gradient of expression has been already described for $W_{n t} 3$ in the dorsal thalamus (Bulfone et al., 1993a). On the other hand, the alternating pattern observed in the hypothalamus between $D l x I$ and $P L Z F$, as previously reported for Otp (Simeone et al., 1994b), supports and stresses the idea that subregionally restricted identities are to be found in the original polyclonal cell lineage of a specific neuromere. Furthermore, alternating stripes betwecn homcobox-containing genes and zinc finger genes are reminiscent of other regulatory genes such as Krox20 and Hoxb-l in the hindbrain transverse neuromeres (Wilkinson et al., 1989a,b; Frohman et al., 1990). In the hindbrain, Krox20 is expressed in rhombomeres 3 and 5, and defines the anterior and posterior borders of the Hoxb-1 expression domain in the rhombomere 4; in our case, $P L Z F$ is expressed along the longitudinal subdomain behind the optic stalk, and $D l x l$ defines its dorsal and ventral borders (Fig. 11; see also Results). Finally, it is interesting to note that $P L Z F$, together with Otp, defines also the alar/basal (longitudinal) boundary of $D l x l$ in the mammillary and retromammillary regions as well as the transverse boundary between the mammillary and the tuberal hypothalamic areas. In the anterior pretectal region, $P L Z F$ is expressed in a transverse segment-like domain. A combined expression analysis with OtxI reveals that $P L Z F$ identifies the anterior pretectal region corresponding to the neuromere D3 described by Figdor and Stern (1993). PLZF is abundantly expressed in this region with a rostral border exactly adjacent to the fasciculus retroflexus and Otxl, also expressed in the anterior pretectum, albeit at lower levels, is strongly expressed in the posterior pretectal area defining the posterior border of $P L Z F$. Furthermore, Pax6 is also segmentally expressed in the posterior pretectum, corresponding to the neuromere D4 (Walther and Gruss, 1991; Figdor and Stern, 1993).

All together, these results strongly support the idea that an intriguing puzzle of regionally restricted genes, possibly belonging to different categories of regulatory genes, is necessary to obtain the correct spatiotemporal information to define segmental and subsegmental identities, by using a sort of highly regulated combinatorial code (Puelles and Rubenstein, 1993; Rubenstein et al., 1994).

As previously shown, $P L Z F$ is also expressed at $\mathrm{E} 13.5$ in a thin stripe corresponding to the zona limitans intrathalamica and at $\mathrm{E} 16.5$ it is still detectable in the lamina medullaris externa. It seems unlikely that $P L Z F$ could be playing a role in the early establishment of this boundary, as it is activated in this region only when morphogenetic boundaries have already been defined 


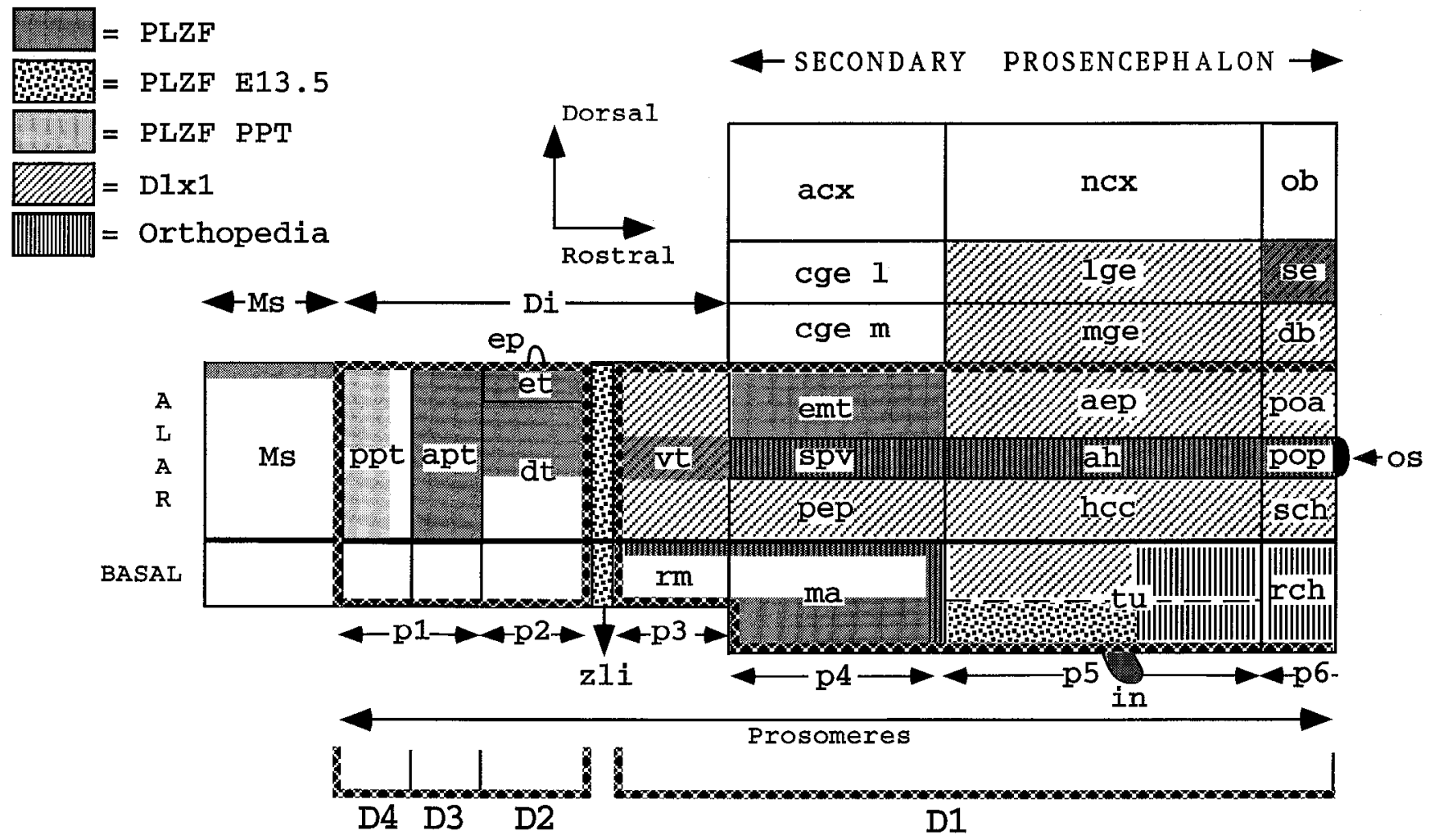

Figure 11. Schematic representation of the $P L Z F$, Otp, and Dlxl expression domains in the transverse and longitudinal subdomains of the CNS regions spanning the mesencephalon, diencephalon, and secondary prosencephalon. On purpose the schematic representation is very similar to that proposed by Bulfone et al. (1993) and Puelles and Rubenstein (1993). However, in the same schente we also included the four diencephalic neuromeres (Dl-D4) deduced by Figdor and Stern (1993). Although the representation and gene expression domains are referred to E 12.5 mouse embryos, we report, as indicated on the top, also the PLZF additional expression domains identified at E 13.5 in the zona limitans intrathalamica and in the posterior basalmost tuberal region. The lower expression of PLZF in the posterior pretectal area is also indicated as a lighter gray. The main longitudinal boundary that separates the alar and basal regions is shown as a ticker horizontal line. Further longitudinal and transverse boundaries that separate transverse neuromeric and longitudinal subdomains are also shown. In this scheme, $P L Z F$ is expressed in predicted defined regions corresponding to longitudinal and transverse regions as well as to segmental boundaries. It is also worth noting the segment-like expression in the anterior pretectal area filling the neuromere D3. This domain of expression, together with those of Otx1 and Pax6 genes in the posterior pretectal area (see Results and Discussion sections), suggests a distinction of the prefectal region in two different transverse domains corresponding to the anterior and posterior pretectal area or to the neuromeres D3 and D4 (Figdor and Stern, 1993). All the abbreviations are explained in the appendix.

and the segmental expression of $D l x l$ sharply identifies the ventral thalamus. However, we suggest that $P L Z F$ might be involved in the maintenance of this boundary or in its cellular differentiation, providing clues to axon pathfinding and patterning.

Later on in the development, $P L Z F$ transcripts are restricted to specific diencephalic nuclei. Several of these nuclei, in particular those in the hypothalamus, could play an important role in the regulation of the vegetative life. One of these, the paraventricular hypothalamic nucleus, releasing neurosecretory effectors, seems to play an important role in the control of several important neurohypophyseal, as well as adenohypophyseal, secretory functions (Kuhlenbeck, 1973). This observation appears particularly interesting considering that $P L Z F$ is strongly expressed in the neurohypophysis and, from E 15 , also in the adenohypophysis, in the thyroid and in the adrenal gland, indicating that $P L Z F$ is a common marker of the major functionally related elements of the endocrine system.

It is also worth considering $P L Z F$ expression in the midbrain, hindbrain, and spinal cord. In all these regions a regionally restricted expression follows the early ubiquitous one and, with the exception of the pons and medulla, $P L Z F$ is preferentially localized to the alar plate, and at E12.5-E 13.5 PLZF is dorsally restricted to the roof plate of the mesencephalon. In the spinal cord, $P L Z F$ is detected in postmitotic cells, localized at the alar boundary between external germinative and mantle layers. In summary, we conclude that $P L Z F$ could play a role in the morphogenetic processes acting to correctly define regional identities within the synencephalon (pretectal area), the posterior parencephalon (dorsal thalamus and epithalamus), the secondary prosencephalon (hypothalamus), as well as, segmentary boundaries between the dorsal and the ventral thalamus and between alar and basal posterior hypothalamus (Fig. 11). Nevertheless, from this analysis additional molecular and morphological evidences emerge supporting the validity of the neuromeric organization of the embryonic mouse forebrain.

\section{Appendix}

List of abbreviations

acx archicortex

aep anterior entopenduncular area

ah anterior hypothalamus

apt anterior pretectum

cge caudal ganglionic eminence (1, lateral; m, medial)

$\mathrm{db}$ diagonal band

D1-D6 neuromeres according to Figdor and Stern (1993) 


$\begin{array}{ll}\text { Di } & \text { diencephalon } \\ \text { dt } & \text { dorsal thalaunus } \\ \text { emt } & \text { eminentia thalami } \\ \text { ep } & \text { epiphysis } \\ \text { et } & \text { epithalamus } \\ \text { hcc } & \text { hypothalamic cell cord } \\ \text { in } & \text { infundibulum } \\ \text { lge } & \text { lateral ganglionic eminence } \\ \text { Ms } & \text { mesencephalon } \\ \text { ma } & \text { mammillary area, hasal zone of p4 } \\ \text { mge } & \text { medial ganglionic eminence } \\ \text { ncx } & \text { neocortex } \\ \text { ob } & \text { olfactory bulb } \\ \text { os } & \text { optic stalk } \\ \text { p1-p6 } & \text { prosomeres 1-6 } \\ \text { pep } & \text { posterior entopendumcular arta } \\ \text { poa } & \text { anterior preoptic area } \\ \text { pop } & \text { posterior preoptic area } \\ \text { ppt } & \text { posterior pretectum } \\ \text { rch } & \text { retrochiasmatic area, basal zone of p6 } \\ \text { rm } & \text { retromammillary area, basal zone of p3 } \\ \text { sch } & \text { suprachiasmatic area } \\ \text { se } & \text { septum } \\ \text { spv } & \text { supraoptic/paraventricular area } \\ \text { tu } & \text { tuberal hypothalamus, basal zone of p5 } \\ \text { vt } & \text { ventral thalamus } \\ \text { zli } & \text { zona limitans intrathalamica } \\ \end{array}$

\section{References}

Altman J, Bayer SA (1978) Development of the diencephalon in the rat. I. Autoradiographic study of the time of origin and settling patterns of neurons of the hypothalamus. J Comp Neurol 182:945-972.

Altman J, Bayer SA (1979) Development of the diencephalon in the rat. IV. Quantitative study of the time of origin of neurons and the internuclear chronological gradients in the thalamus. J Comp Neurol 188:455-472.

Altman J, Bayer SA (1986) The development of the rat hypothalamus. Adv Anat Embryol Cell Biol 100:1-177.

Altman J, Bayer SA (1988) Development of the rat thalamus: I. Mosaic organization of the thalamic neuroepithelium. J Comp Neurol 275: 346-377.

Bardwell VJ, Treisman R (1994) The POZ domain: a conserved protein-protein interaction motif. Genes Dev 8:1664-1677.

Bulfone A, Puelles L, Porteus MH, Frohman MA, Martin GR, Rubenstein JLR (1993a) Spatially restricted expression of $D l x-1, D l x-2$ (Tes-1), Gbx-2, and Writ-3 in the embryouic day 12.5 mouse forebrain defines potential transverse and longitudinal segmental boundaries. $\mathrm{J}$ Neurosci 13:3155-3172.

Bulfone A, Kim H-J, Puelles L, Porteus MH, Grippo JF Rubenstein JLR (1993b) The mouse $D l x-2($ Tes-l) gene is expressed in spatially restricted domains of the forebrain, face and limbs in midgestation mouse embryos. Mech Dev 40:129-140.

Chen Z, Brand NJ, Chen A, Chen S-J, Tong J-H, Wang Z-Y, Waxman S, Zelent A (1993) Fusion between a novel Krüppel-like zinc finger gene and the retinoic acid receptor-a locus due to a variant $t(11$; 17) translocation associated with acute promyelocytic leukaemia. EMBO J 12:1161-1167.

Cohen SM (1990) Specification of limb development in the Drosophila embryo by positional cues from segmentation genes. Nature 343: 173-177.

Cohen S, Jürgens G (1991) Drosophila headlines. Trends Genet 7:267272.

Conlon RA, Rossant J (1992) Exogenous retinoic acid rapidly induces anterior ectopic expression of murine Hox-2 genes in vivo. Development 116:357-368

Dalton D, Chadwick R, McGinnis W (1989) Expression and embryonic function of empty spiracles: a Drosophila homeobox gene with two patterning functions on the anterior-posterior axis of the embryo. Genes Dev 3:1940-1956.

Figdor MC, Stern CD (1993) Segmental organization of embryonic diencephalon. Nature 363:630-634.

Finkelstein R, Perrimon N (1991) The molecular genetics of head development in Drosophila melanogaster. Development 112:899-912.

Fraser S, Keynes R, Lumsden A (1990) Segmentation in chick embryo hindbrain is defined by cell lineage restrictions. Nature 344:431-435.
Frohman MA, Boyle M, Martin G (1990) Isolation of the mouse Hox2.9 gene: analysis of embryonic expression suggest that positional information along the anteroposterior axis is specified by mesoderm. Development 1 10:589-607.

Gallera J (1971) Primary induction in birds. Adv Morphol 9:149-180.

Gurdon JB (1987) Embryonic induction-molecular prospects. Development 99:285-306.

Hara K (1978) Spemann's organiser in birds. In: Organiser-a milestone of a half-century since Spemann (Nakamura $\mathrm{O}$, Toivonen $\mathrm{S}$, eds), pp 221-265. Amsterdam: Elsevier.

Herrick CJ (1933) Morphogenesis of the brain. J Morphol 54:233-258.

Hogan BLM, Thaller C, Eichele G (1992) Evidence that Hensen's node is a site of retinoic acid synthesis. Nature 359:237-241.

Jessell TM, Melton AD (1992) Diffusible factors in vertebrate embry onic induction. Cell 68:257-270.

Kim Y, Nirenberg M (1989) Drosophila NK-homeobox genes. Proc Natl Acad Sci USA 86:7716-7720.

Kuhlenbeck H (1973) The central nervous system of vertebrates. Basel: Karger.

Lumsden A (1990) The cellular basis of segmentation in the developing hindbrain. Trends Neurosci 13:329-335.

Lumsden A, Keynes R (1989) Segmental patterns of neuronal development in the chick hindbrain. Nature 337:424-428.

Marshall H, Nonchev S, Sham MH, Muchamore I, Lumsden A, Krumlauf R (1992) Retinoic acid alters hindbrain Hox code and induces transformation of rhombomeres $2 / 3$ into $4 / 5$ identity. Nature 360 : 737-741.

Martinez S. Wassef M, Alvarado-Mallart R-M (1991) Induction of a mesencephalic phenotype in the 2-day-old chick prosencephalon is preceded by the early expression of the homeobox gene en. Neuron 6:971-981.

McGinnis W, Krumlauf R (1992) Homeobox genes and axial patterning. Cell 68:283-302.

McMahon AP, Joyner AL, Bradley A, McMahon JA (1992) The midbrain-hindbrain phenotype of Wnt-1/Wnt-1 mice results from stepwise deletion of engrailed-expressing cells by 9.5 days postcoitum. Cell 69:581-595.

Porteus MH, Bulfone A, Ciaranello RD, Rubenstein JLR (1991) Isolation and characterization of a novel cDNA clone encoding a homeodomain that is developmentally regulated in the ventral forebrain. Neuron 7:221-229.

Price M, Lemaistre M, Pischetola M, Di Lauro R, Duboule D (1991) A mouse gene related to distal-less shows a restricted expression in the developing forebrain. Nature 351:748-751.

Price M, Lazzaro D, Pohl T, Mattei MG, Rüther U, Olivo J-C, Duboule D, Di Lauro R (1992) Regional expression of the homeobox gene $N k x-2.2$ in the developing mammalian forebrain. Neuron 8:241-255.

Puelles L, Rubenstein JLR (1993) Expression patterns of homeobox and other putative regulatory genes in the embryonic mouse forebrain suggest a neuromeric organization. Trends Neurosci 16:472-479.

Puelles L, Amat JA, Martinez del la Torre M (1987) Segment-related, mosaic neurogenetic pattern in the forebrain and mesencephalon of early chick embryos: I. Topography of AChE-positive neuroblasts up to stage HH18. J Comp Neurol 266:247-268.

Roelink H, Nusse R (1991) Expression of two members of the Wnt family during mouse development-restricted temporal and spatial patterns in the developing neural tube. Genes Dev 5:381-388.

Rubenstein JLR, Martinez S, Shimamura K, Puelles L (1994) The embryonic vertebrate forebrain: the prosomeric model. Science 266 $578-580$.

Ruiz i Altaba A (1993) Induction and axial patterning of the neural plate: planar and vertical signals. J Neurobiol 24:1276-1304.

Ruiz i Altaba A (1994) Pattern formation in the vertebrate neural plate. Trends Neurosci 17:233-243.

Saiki R, Gelfand D, Stoffel S, Scharf S, Higuchi R, Horn G, Mullis K, Elrich H (1988) Primer-directed enzymatic amplification of DNA with a thermostable DNA polymerase. Science 238:487-494

Sambrook J, Fritsch EF Maniatis T (1989) Molecular cloning. A laboratory manual, 2d ed. Cold Spring Harbor, NY: Cold Spring Harbor Laboratory.

Simeone A, Acampora D, Arcioni L, Andrews PW, Boncinelli E, Mavilio F (1990) Sequential activation of the HOX2 homeobox genes by retinoic acid in human embryonal carcinoma cells. Nature 346: $763-766$

Simeone A, Acampora D, Nigro V, Faiella A, D'Esposito M, Stornai- 
uolo A, Mavilo F, Boncinelli E (1991) Differential regulation by retinoic acid of the homeobox genes of the four Hox loci in human embryonal carcinoma cells. Mech Dev 33:215-228.

Simeone A, Gulisano M, Acampora D, Stornaiuolo A, Rambaldi M, Boncinelli E (1992a) Two vertebrate homeobox genes related to the Drosophila empty spiracles gene are expressed in the embryonic cerebral cortex. EMBO J 11:2541-2550.

Simeone A, Acampora D, Gulisano M, Stornaiuolo A, Boncinelli E (1992b) Nested expression domains of four homeobox genes in developing rostral brain. Nature 358:687-690.

Simeone A, Acampora D, Mallamaci A, Stornaiuolo A, D'Apice MR, Nigro V, Boncinelli E (1993) A vertebrate gene related to orthodenticle contains a homeodomain of the bicoid class and demarcates anterior neuroectoderm in the gastrulating mouse embryo. EMBO J 12:2735-2747.

Simeone A, Acampora D, Pannese M, D'Esposito M, Stornaiuolo A, Gulisano M, Mallamaci A, Kastury K, Druck T, Huebner K, Boncinelli $E$ (1994a) Cloning and characterization of two members of the vertebrate $D l x$ gene family. Proc Natl Acad Sci USA 91:2250-2254.

Simeone A, D'Apice MR, Nigro V, Casanova J, Graziani G, Acampora D, Avantaggiato V (1994b) Orthopedia, a novel homeobox-containing gene expressed in the developing central nervous system of both mouse and Drosophila. Neuron 13:83-101.

Smith JC (1994) Hedgehog, the floor plate and the zone of polarizing activity. Cell 76:193-196.

Spemann H, Mangold H (1924) Über induktion von Embryonanlagen durch Implantation artfremder Organisatoren. Wilhelm Rouxs Arch EntwMech Organ 100:599-638.
Stoykova A, Gruss P (1994) Roles of Pax-genes in developing and adult brain as suggested by expression patterns. J Neurosci 14:13951412 .

Storey KG, Crossley JM, De Robertis EM, Norris WN, Stern CD (1992) Neural induction and regionalisation in the chick embryo. Development 114:729-741.

Thaller C, Eichele G (1987) Identification and spatial distribution of retinoids in the developing chick limb bud. Nature 327:625-628.

Thaller C, Eichele G (1990) Isolation of 3,4-didehydroretinoic acid, a novel morphogenetic signal in the chick wing bud. Nature 345:815819.

Vaage $S$ (1969) The segmentation of the primitive neural tube in chick embryos (Gallus domesticus). Adv Anat Embryol Cell Biol 41:1-87.

Wagner M, Thaller C, Jessell T, Eichele G (1990) Polarizing activity and retinoid synthesis in the floor plate of the neural tube. Nature 345:819-822

Wagner M, Han B, Jessell TM (1992) Regional differences in retinoid release from embryonic neural tissue detected by an in vitro reporter assay. Development 116:55-66.

Walther C, Gruss P (1991) Pax-6, a murine paired box gene, is expressed in the developing CNS. Development 113:1435-1449.

Wilkinson D, Bhatt S, Cook M, Boncinelli E, Krumlauf R (1989a) Segmental expression of Hox-2 homeobox-containing genes in the developing mouse hindbrain. Nature 341:405-409.

Wilkinson DG, Bhatt S, Chavrier P, Bravo R, Charnay P (1989b) 2Segment-specific expression of a zinc-finger gene in the developing nervous system of the mouse. Nature 337:461-464. 